\title{
ARTICLE OPEN Sustained Type I interferon signaling as a mechanism of resistance to PD-1 blockade
}

\author{
Nicolas Jacquelot ${ }^{1,2,3,4,26}$, Takahiro Yamazaki ${ }^{1,3}$, Maria P. Roberti (iD ${ }^{1,3,5}$, Connie P. M. Duong (D) ${ }^{1,3}$, Miles C. Andrews (iD) $6,7,8$, \\ Loic Verlingue $^{3,9}$, Gladys Ferrere ${ }^{1,3,5}$, Sonia Becharef ${ }^{1,3}$, Marie Vétizou ${ }^{1,3}$, Romain Daillère ${ }^{1,3}$, Meriem Messaoudene ${ }^{1,3}$, \\ David P. Enot ${ }^{3,10}$, Gautier Stoll ${ }^{10,11,12,13,14}$, Stefano Ugel ${ }^{15}$, Ilaria Marigo ${ }^{16}$, Shin Foong Ngiow ${ }^{17,18}$, Aurélien Marabelle ${ }^{1,2,3}$, \\ Armelle Prevost-Blondel (iD) ${ }^{14,19,20}$, Pierre-Olivier Gaudreau ${ }^{6}$, Vancheswaran Gopalakrishnan ${ }^{6}$, Alexander M. Eggermont ${ }^{3}$, Paule Opolon ${ }^{3}$, \\ Christophe Klein ${ }^{11}$, Gabriele Madonna ${ }^{21}$, Paolo A. Ascierto $\mathbb{D}^{21}$, Antje Sucker ${ }^{22}$, Dirk Schadendorf $\mathbb{D}^{22}$, Mark J. Smyth (iD) ${ }^{17,18}$, \\ Jean-Charles Soria ${ }^{2,3,4,9}$, Guido Kroemer $\mathbb{I D}^{2,3,4,10,11,12,13,14,23,24}$, Vincenzo Bronte ${ }^{15}$, Jennifer Wargo $\mathbb{( \mathbb { D }}^{6,25}$ and Laurence Zitvogel ${ }^{1,2,3,4,5}$
}

PD-1 blockade represents a major therapeutic avenue in anticancer immunotherapy. Delineating mechanisms of secondary resistance to this strategy is increasingly important. Here, we identified the deleterious role of signaling via the type I interferon (IFN) receptor in tumor and antigen presenting cells, that induced the expression of nitric oxide synthase 2 (NOS2), associated with intratumor accumulation of regulatory T cells (Treg) and myeloid cells and acquired resistance to anti-PD-1 monoclonal antibody $(\mathrm{mAb})$. Sustained IFN $\beta$ transcription was observed in resistant tumors, in turn inducing PD-L1 and NOS2 expression in both tumor and dendritic cells (DC). Whereas PD-L1 was not involved in secondary resistance to anti-PD-1 mAb, pharmacological or genetic inhibition of NOS2 maintained long-term control of tumors by PD-1 blockade, through reduction of Treg and DC activation. Resistance to immunotherapies, including anti-PD-1 mAb in melanoma patients, was also correlated with the induction of a type I IFN signature. Hence, the role of type I IFN in response to PD-1 blockade should be revisited as sustained type I IFN signaling may contribute to resistance to therapy.

Cell Research (2019) 29:846-861; https://doi.org/10.1038/s41422-019-0224-x

\section{INTRODUCTION}

Major conceptual advances in cancer biology have been made over the past decade. ${ }^{1-3}$ The understanding that immune responses are routinely generated against tumor-specific neoantigens resulting from cancer-associated mutations ${ }^{4-8}$ and commonly suppressed by immunosuppressive tumor microenvironments (TME) has led to the development of effective immunotherapies aimed at provoking immune control against tumor progression. ${ }^{9,10}$ Cancer immunotherapy has resulted in remarkable success in the treatment of a variety of hematological and solid metastatic malignancies such as melanoma, lung, bladder, kidney, Hodgkin's lymphoma, B cell acute lymphocytic leukemia, hepatocellular carcinoma, Merkel-cell carcinoma and head and neck tumors. ${ }^{1-16}$ To date, therapies that block inhibitory signaling molecules expressed by $\mathrm{T}$ lymphocytes (so called "immune checkpoints") during the initial priming phase (in the draining lymph nodes) or the effector phases (in tumor beds) of adaptive anticancer immune responses have resulted in the greatest clinical benefit. ${ }^{16,17} \mathrm{~A}$ paradigm of this success has been the use of monoclonal antibodies (mAb) targeting Programmed cell death 1 (PD-1) (expressed by activated/exhausted T cells) or its ligand PD-L1 (commonly expressed by cancer cells or cells of the TME). ${ }^{16,18}$ By releasing these molecular brakes, such mAbs reinstate the anticancer adaptive arm of the immune response.

While PD-1 blockade represents the most effective first line therapy in BRAF wildtype melanoma and the best option in first-

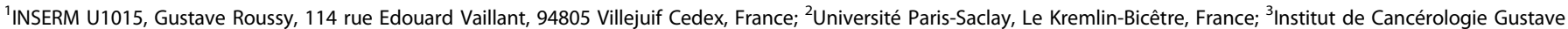

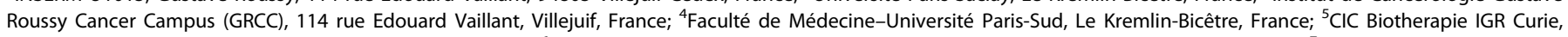

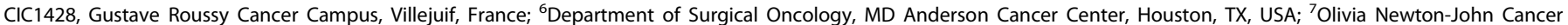

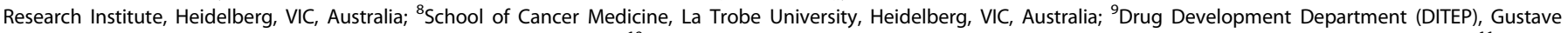

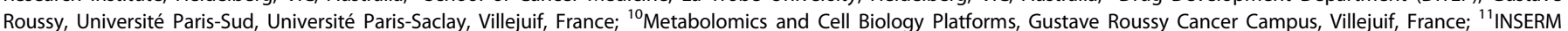

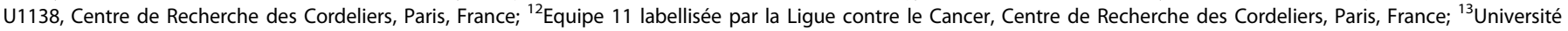

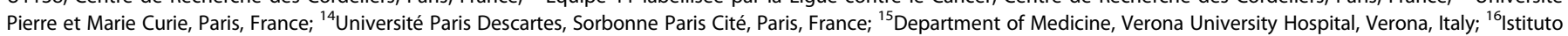

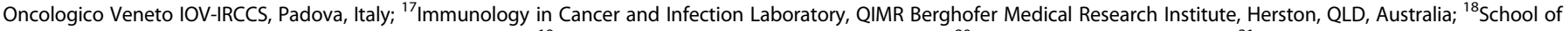

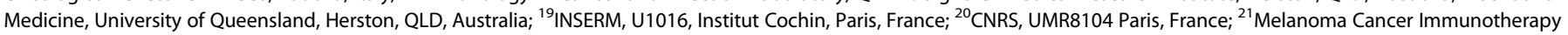

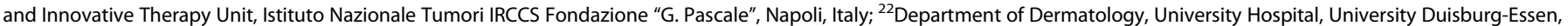

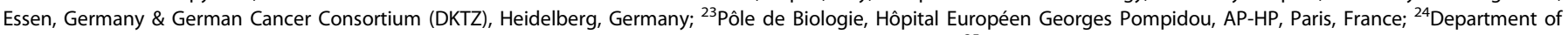

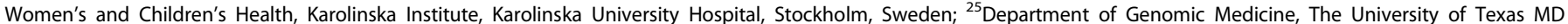
Anderson Cancer Center, Houston, TX, USA and ${ }^{26}$ Present address: Immunology Division, The Walter and Eliza Hall Institute of Medical Research, Melbourne, VIC, Australia Correspondence:

Guido Kroemer (kroemer@orange.fr) or Laurence Zitvogel (Laurence.ZITVOGEL@gustaveroussy.fr)

These authors contributed equally: Nicolas Jacquelot, Takahiro Yamazaki, Maria P. Roberti

Received: 5 March 2019 Accepted: 5 August 2019

Published online: 3 September 2019 
line non-small cell lung cancer, when combined with platinumbased chemotherapy, about $60-70 \%$ of tumors do not clinically benefit from this treatment and exhibit primary resistance to this therapeutic strategy. ${ }^{19,20}$ Primary resistance has been attributed to several factors including low tumor mutational burden and poor intrinsic antigenicity of tumor cells ${ }^{5,6}$ defective antigen presentation and priming phase; ${ }^{21}$ limited tumor infiltration related to exhausted T cell functions; ${ }^{2}$ CSF1-dependent tumor associated macrophage accumulation; ${ }^{22}$ and immunosuppressive metabolic pathways related to adenosine and indoleamine-2,3-dioxygenase (IDO). ${ }^{2}$ Importantly, genomic defects in IFNץ signaling pathway genes have been found to provide a primary mechanism leading to resistance to therapy targeting cytotoxic T-lymphocyteassociated protein 4 (CTLA-4), including in melanoma. ${ }^{23}$

More recently, specific mechanisms of secondary resistance to chronic inhibition of PD-1 receptors have been described in about $25 \%$ of melanoma patients. ${ }^{24-26}$ A subset of melanoma patients who progressed despite an initial response to therapy with pembrolizumab, which targets PD-1, displayed either loss-offunction mutations in Janus kinases JAK1 or JAK2, leading to reduced sensitivity to the anti-proliferative effects of IFNs, decreased phosphorylation of STAT1, or a truncating mutation in the gene $\beta 2$ microglobulin, resulting in defective antigen presentation due to prevention of folding and transport of MHC class I molecules to the cell surface for T cell recognition of tumor cells. ${ }^{26}$ Similar alterations in $\beta 2$ microglobulin have been found in about $30 \%$ of non-responders treated with a combination of antiPD-1 and anti-CTLA-4. ${ }^{27}$ In addition to the natural selection of heritable genetic (or epigenetic) traits previously described, ${ }^{28,29}$ other acquired resistance mechanisms have also been reported such as the IFN $\gamma$-inducible expression of PD-L1; $;^{30,31}$ TNFa-induced loss of antigenic variants; ${ }^{32}$ and TCR-dependent upregulation of additional exhaustion markers on activated $\mathrm{T}$ lymphocytes including T-cell immunoglobulin and mucin-domain containing3 (Tim3), lymphocyte activation gene 3 (Lag3), T cell immunoreceptor with Ig and ITIM domains (TIGIT), B and T cell lymphocyte attenuator (BTLA), and V-domain Ig suppressor of $\mathrm{T}$ cell activation (VISTA). ${ }^{2,11,24}$

Prompted by the relatively short duration of tumor control following iterative administrations of anti-PD- $1 \mathrm{mAb}$ in tumor bearing mice, we identified a detrimental role of interferon- $\alpha / \beta$ receptor (IFNAR) signaling in both $\mathrm{CD}_{4} 5^{-}$and $\mathrm{CD} 45^{+}$intratumoral subsets inducing the expression of nitric oxide synthase 2 (NOS2). This was associated with the reduction of intratumor polyfunctional cytotoxic T lymphocytes (CTLs), increased regulatory $T$ cells and myeloid cells leading to acquired resistance to anti-PD-1 mAb-based therapy. A chronic PD-1 blockade progressively induced IFN $\gamma$ and IFN $\beta$ transcription in the TME, with both IFNs in turn triggering PD-L1 and NOS2 expression on both tumor cells and leukocytes. Anti-PD-1 mAb-sensitive tumors exhibited lower endogenous levels of NOS2, and progressively lost TME-derived IFN $\beta$ production. In contrast, resistant tumors, presenting high NOS2 expression at baseline, sustained their IFN $\beta$ release, the main trigger of NOS2 upregulation and maintenance. While PD-L1 was not involved in secondary resistance to anti-PD-1 mAb, reducing NOS2 with L-NAME or a genetic knockout improved long-term tumor control by PD-1 blockade in several tumor models. Finally, we highlight the potential clinical significance of this pathway in secondary resistance to first-line therapies (including PD-1 blockade) in advanced melanoma patients.

\section{RESULTS}

Resistance of murine tumors to PD- 1 blockade

As resistance to anti-PD-1 treatment is a major clinical issue, a better understanding of the cellular and molecular mechanisms involved is critical. While, many mechanisms have been described to be responsible for primary resistance, ${ }^{2,5,6,21,22}$ the mechanisms underlying secondary or acquired resistances to anti-PD-1 therapies are still not well understood. Secondary or acquired resistance to anti-PD-1 blockade is defined as a loss of treatment efficacy while being on therapy, resulting in tumor growth and progression. To tackle this question, we treated four established transplantable tumor models syngeneic to C57BL/6 mice with 4 biweekly intraperitoneal (i.p.) administrations of anti-PD-1 mAb (clone RMP1-14) (Fig. 1a). While the MCA205WT sarcoma remained sensitive to 4 doses of PD-1 blockade over 12 days (Fig. 1b; Supplementary information, Fig. S1b), hosts bearing MCA205OVA and MC38 colon cancers were eventually resistant as initial treatments slightly reduced their tumor growth kinetics (Fig. 1c; Supplementary information, Fig. S1a, c and e). However, AT3 breast tumors were primarily resistant to $m A b$, exhibiting no benefit in tumor control or overall survival (Fig. 1d; Supplementary information, Fig. S1d). ${ }^{33}$ Whilst tumor growth in the sensitive (MCA205WT) model displayed a rebound after the period of active anti-PD-1 therapy, we showed that prolonging the administration of anti-PD-1 mAb from 4 to 6 doses failed to extend the efficacy of therapy, culminating in overt tumor progression in the majority of animals (Fig. 1e, f; Supplementary information, Fig. S1f). However, concurrent CTLA-4 blockade in this model could prevent secondary resistance to this treatment (Fig. 1e, f).

We then performed a comprehensive immunological phenotyping of splenocytes and intratumor cells (Supplementary information, Fig. S2a, b) after 4 administrations of anti-PD-1 mAb in sensitive (MCA205WT sarcoma), eventually resistant (MCA205OVA sarcoma) and innately resistant (AT3 breast cancer) tumor models. Surrogate hallmarks of anticancer efficacy were observed consistently in all treated mice bearing MCA205WT but only selectively in responding mice harboring MCA205OVA cells reflected by intratumor leukocyte influx, the upregulation of ICOS expression on $\mathrm{CD}^{+}$TILs-relative to anti-PD-1 non-responders (NR) in MCA2050VA mice-the increase in polyfunctional $\left(\right.$ IFN $\left.\gamma^{+} \mathrm{TNFa}^{+}\right) \mathrm{CTLS}$ and an increased $\mathrm{CD}^{+} / \mathrm{CD}^{+}{ }^{+} \mathrm{FOXP3}^{+}$ratio (in MCA205 only) (Supplementary information, Fig. S2c, d). Splenic immune parameters failed to correlate with the sustained success of PD-1 blockade (Supplementary information, Fig. S2a).

Hence, we took advantage of the contrast between relatively sensitive MCA205WT (although showing a late escape from the PD-1 blockade) and MC38 tumors that promptly overcome PD-1 blockade to scrutinize the underlying mechanisms of delayed resistance to PD-1 blockade.

Interrogation of host and tumor IFNAR signaling pathways in resistance to PD-1 blockade

A large body of literature strongly supports the key role of the IFNAR signaling pathway in controlling anticancer immunosurveillance, and dictating the success of chemotherapy and radiotherapy-induced immune responses, even against MHC class I-deficient tumors that are innately resistant to PD-1 blockade. ${ }^{34-37}$ The expression of IFNAR1 was comparable between the MCA205, MC38 and AT3 tumor cell lines (Supplementary information, Fig. S3). They were all sensitive to in vitro stimulation with IFNa or IFN $\gamma$, resulting in MHC class I and/or PD-L1 upregulation, as well as CXCL10 secretion in all cell lines at least initially sensitive to PD-1 inhibition (Supplementary information, Fig. S3). We next investigated the role of IFNAR1 expressed by the host or intrinsically by tumor cells in affecting the sensitivity of MCA205WT tumors to anti-PD-1 mAb in vivo. While we saw no differences in tumor control based on IFNAR1 status in the absence of treatment, administration of anti-PD-1 mAb into Ifnar $^{-1-}$ mice extended tumor control and prolonged survival compared with that observed in wild type (WT) mice (Fig. 2a). Accordingly, the surrogate hallmarks of efficacy of PD-1 blockade were all markedly enhanced in the absence of a functional IFNAR1 host signaling pathway (Fig. 2b). Whilst the intratumor fraction of $\mathrm{CD} 45^{+}$cells 

$+$

$\downarrow \downarrow \downarrow \downarrow$

Tumor growth monitoring

\section{Tumor size}

$20-25 \mathrm{~mm}^{2}$

b
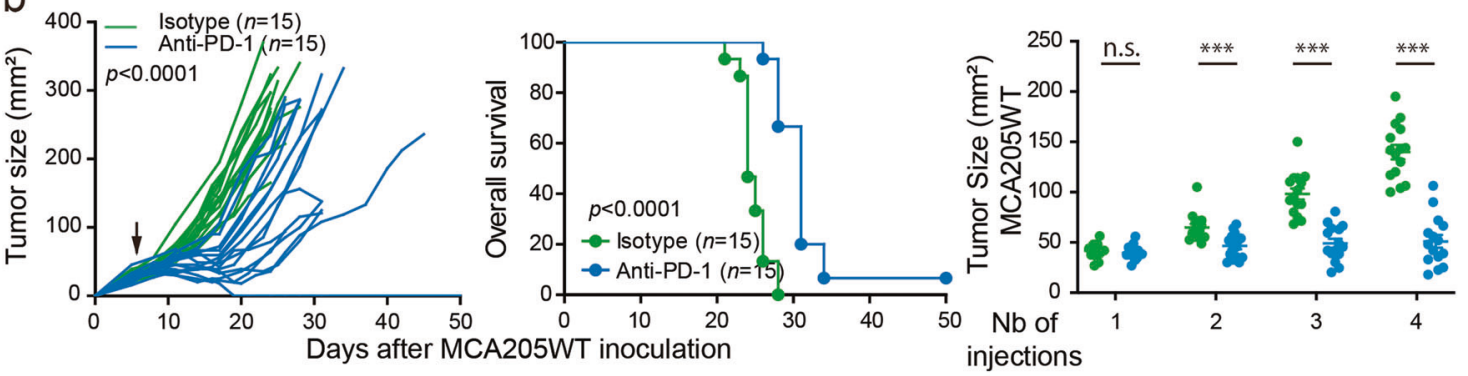

C
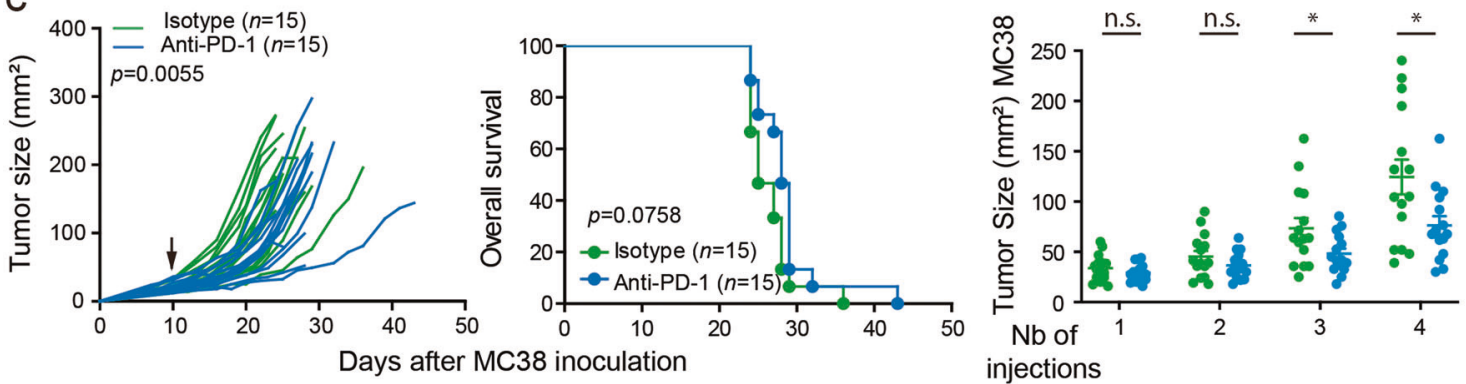

d
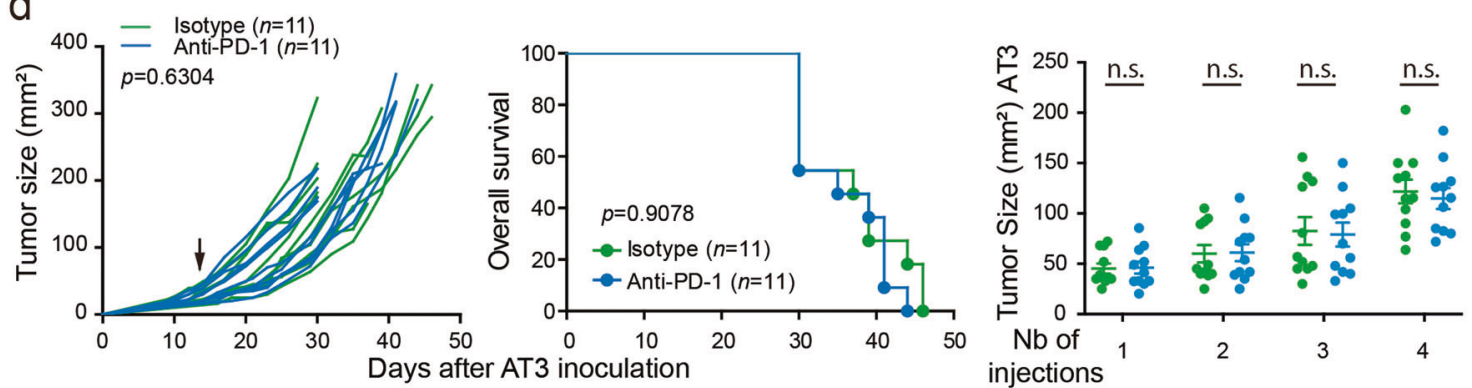

e

Day 0

Anti-PD-1/Anti-CTLA-4 or Isotype controls

Tumor inoculation

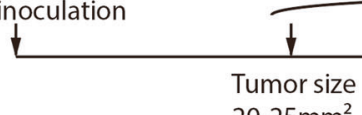

$20-25 \mathrm{~mm}^{2}$

$f$

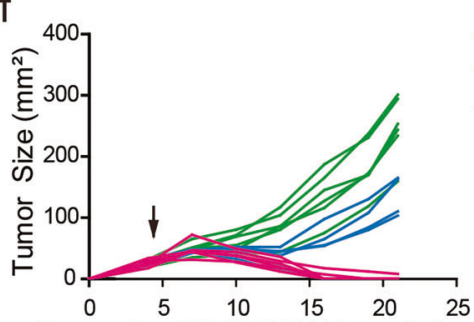

Days after MCA205WT inoculation
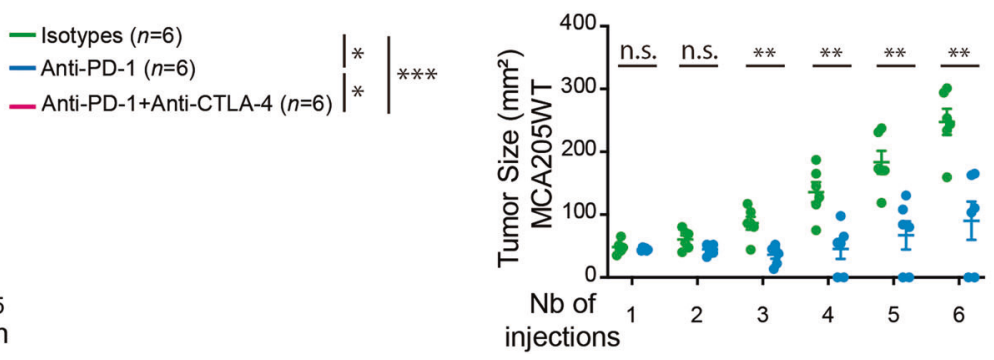

was not significantly increased, augmented ICOS-expressing CTLS, together with reduced Treg infiltration and an increased $\mathrm{CD}^{+}$/ $\mathrm{CD}^{+}{ }^{\mathrm{FOXP}} 3^{+}$ratio, were observed in $/$fnar $^{-l-}$ compared with Ifnar $1^{+/+}$anti-PD-1-treated mice (Fig. 2c). Moreover, the therapeutic outcome of a PD-1 blockade was influenced by tumor cell expression of IFNAR1. In fact, two MCA205 clones,19-14 and
$19-37,{ }^{38}$ produced by nucleo-transfection with specific zinc finger nucleases (ZFN) causing targeted deletion of the Ifnar1 gene were implanted into Ifnar 1 wild-type mice. These exhibited inherently reduced tumor growth kinetics and, more importantly, heightened response to anti-PD-1 mAb resulting in complete tumor eradication in up to $17 \%$ of mice (Fig. 2 d). 
Fig. 1 "Sensitive", "eventually resistant" and "innately resistant" tumor models to PD-1 blockade. a and e Therapeutic antitumor protocols based on anti-PD-1 or its isotype control. When tumors reached $20-25 \mathrm{~mm}^{2}$ (indicated by an arrow), anti-PD-1 mAb (or its isotype control) alone a or together with anti-CTLA-4 mAb e were administered i.p. every 3 days for 4 a to 6 e injections (as described in Material and Methods). b-d, f MCA205WT sarcoma b, f, MC38 colon c or AT3 breast d cancer cells were injected subcutaneously. Tumor growth kinetics (left panels), survival curves (middle panels) and tumor sizes after sequential injections of isotype or anti-PD-1 mAb (right panels) are depicted. Each line or dot corresponds to one animal. Each graph represents 1 experiment $\mathbf{f}$, a pool of $2 \mathbf{d}$ to $3 \mathbf{b}$, c experiments with 5-6 animals per group and per experiment. For tumor growth and Kaplan-Meier curves, statistical analyses were performed using the specific software detailed in the Material and Methods. Unpaired t-tests were used in b, $\mathbf{c}$, d and f, right panels. ${ }^{*} p<0.05,{ }^{* *} p<0.01,{ }^{* * *} p<0.001, n . s .:$ not significant. Means \pm SEM are represented

To understand the autocrine regulation of type I IFN release by either tumor or host cells, we subjected tumor cell lines and bone marrow-derived myeloid cells (BMMCs) or bone-marrow derived dendritic cells (BMDCs) to a 24-h stimulation with recombinant IFNa. The relatively anti-PD-1-resistant MC38 and AT3 cells significantly upregulated Ifn $\beta 1$ transcription in response to IFNa whilst no significant change was observed in the anti-PD-1sensitive MCA205WT or Ifnar $1^{-1-}$ sarcoma clones (Fig. 3a). Consistent with other reports, ${ }^{34-36}$ BMDCs exhibited positive feedback of type I IFN expression in an IFNAR1-dependent manner, in contrast to BMMCs (Fig. 3b). Along the course of PD1 blockade, we observed that both intratumor $\mathrm{CD} 45^{+}$and $\mathrm{CD} 45^{-}$ cell subsets sorted from sensitive tumors (MCA205WT) tended to lose the expression of some interferon-stimulated gene (ISG) gene products in vivo (Fig. 3c-e; Supplementary information, Fig. $\mathrm{S} 4 \mathrm{a}-\mathrm{C}$ ). This was in contrast to resistant tumors (MC38) that tended to maintain Ifn $\beta 1, M x 1$ and Isg15 gene expression (Fig. 3f-h; Supplementary information, Fig. S4d-f).

Taken together, the IFNAR1 signaling pathways comes into play in tumor cells and leukocytes to subvert tumor growth control by anti-PD-1 mAb, especially when type I IFNs are produced by both $\mathrm{CD}_{4} 5^{+}$and $\mathrm{CD} 45^{-}$cellular compartments.

PD-1 blockade broadly induced PD-L1 upregulation with no influence on tumor progression

To further explore the effect of PD-L1 expression in cancer resistance to IFN $\gamma$ producing CTLs that express PD-1, ${ }^{31}$ we investigated the regulation of PD-L1 transcription in all tumor cell lines by qRT-PCR in vitro. All cell lines tended to upregulate PdI1 gene transcription similarly after IFNץ stimulation, while type I IFN stimulation was IFNAR1-dependent (Supplementary information, Fig. S5a). We further analyzed the expression of PD-L1 on the cell surface of tumor and immune cells in sensitive MCA205WT or resistant AT3 tumors after 4 injections using flow cytometry (Supplementary information, Fig. S5b-e). As expected, both tumor infiltrating myeloid cells and the $\mathrm{CD} 45^{-}$fraction acquired PD-L1 expression. Cell subsets from sensitive tumors expressed much higher PD-L1 levels than those from resistant tumors (Supplementary information, Fig. S5b-d). The expression of PD-L1 on CD45 $5^{-}$cells was more pronounced in Ifnar1-deficient hosts and this was associated with improved anti-PD-1 therapeutic efficacy (Fig. 2a; Supplementary information, S5e).

Despite these observations, four lines of experimental evidence underscored the lack of functional relevance of PD-L1 upregulation in secondary resistance to anti-PD-1 mAb. First, concomitant or sequential co-blockade of PD-1 and PD-L1 receptor/ligand failed to improve tumor control (Fig. 4a). Second, the anti-PD-1 $\mathrm{mAb}$ did not exhibit more sustained antitumor effects against three independent clones of PD-L1-deficient sarcoma (Fig. 4b). Third, co-inhibition of PD-1 and CD80, the alternate receptor for PD-L1, using anti-CD80 mAb or a CTLA-4-lg fusion protein, Abatacept, failed to control tumor escape or prolong survival over PD-1 blockade alone (Fig. 4c; Supplementary information, Fig. S6). Finally, the significant inverse correlation observed between tumor size and endogenous levels of PD-L1 expression by CD45 cells as measured by MFI, in the absence of therapy (Supplementary information, Fig. S6b), was not seen following treatment with
anti-PD-1 (Supplementary information, Fig. S6c). This suggested that PD-L1 expression was more likely to be an indirect hallmark of natural anticancer immunosurveillance, rather than a surrogate marker of secondary resistance of PD-1 blockade. Additionally, this anti-correlation was also not observed in Ifnar1-deficient tumor clones (not shown), corroborating the intrinsic role of type I IFN in natural immunosurveillance. ${ }^{39}$

These data suggest that type I IFN contributes to the upregulation of PD-L1 expression in the TME during the course of anti-PD-1 therapy, likely reflecting the extent of underlying adaptive anticancer immune responses. However, PD-L1 upregulation per se is unlikely to be the cause of secondary resistance to PD-1 blockade in our pre-clinical model.

\section{IFNs-induced NOS2 compromises sustained responses to PD-1 blockade}

The type I and type II IFN receptor signaling pathways culminate in ISRE and GAS-inducible promoter transcriptional activity of various ISG including the well-described inducible nitric oxide synthase (Nos2) gene product. ${ }^{40-42}$ We contrasted a microarray analysis of all protein-coding transcripts expressed in $\mathrm{CD} 45^{+}$fractions of established MC38 tumors after 1 and 2 administrations of isotype control versus blocking anti-PD-1 mAb (Fig. 5a, Supplementary information, Table S1). Nos2 was the third most upregulated gene product after PD-1 blockade in TILs (Table S1). We confirmed these findings by $\mathrm{qRT}-\mathrm{PCR}$ on the $\mathrm{CD} 45^{+}$fraction, which showed a prompt upregulation of Nos2 expression in PD-1 treated tumors $48 \mathrm{~h}$ after the 1st injection (Fig. 5b), preceding the clinical observation of anti-PD-1 resistance (Fig. 1c; Supplementary information, S2c). This upregulation was not observed in the CD45 ${ }^{-}$fraction (not shown). In MCA205WT, Nos2 was significantly upregulated after 3 anti-PD-1 injections (Fig. 5c) and this, once again, preceded the therapy resistance observed after 4 injections (Fig. 1b, 1f; Supplementary information, Fig. S1f). Surprisingly, Nos2 expression tended to decrease after 4 injections (Fig. 5c). However, at the protein level, flow cytometry analyses performed after 4 injections of $\mathrm{mAb}$ revealed two-fold higher NOS2 expression in $\mathrm{CD}_{11 \mathrm{C}^{+}}$as well as $\mathrm{F} 4 / 80^{+} / \mathrm{Gr} 1^{-}$fractions of $\mathrm{CD}^{+} 5^{+}$tumor infiltrating cells from anti-PD-1-treated mice compared with isotype-treated controls (Fig. 5d). qRT-PCR and flow cytometric analyses showed that type I IFN (or LPS)-stimulated BMDCs expressed higher levels of Nos2 than BMMCs (Fig. 5e; Supplementary information, Fig. S7). Tumor cells upregulated Nos2 expression in response to type I or II IFN stimulation in vitro (Fig. 5f). In addition, the CD45 ${ }^{-}$fraction from MCA205WT tumors in vivo also expressed higher levels of Nos2 at later stages of PD-1 blockade (Fig. $5 \mathrm{~g}$ ) with a trend towards higher NOS2 protein levels in mAb-treated tumor cells compared with isotype (Fig. 5h). In contrast, the neuronal Nos 1 and the endothelial Nos3 gene transcripts were not significantly regulated by type I IFNs, in either tumor cells or leukocytes (Supplementary information, Fig. S8). We also investigated the expression of Arginase $1(\operatorname{Arg} 1)$, which is known to be involved in immunosuppressive functions as a second pathway regulating the arginine availability in the TME. ${ }^{43,44}$ Contrary to Nos2, type 2 cytokines IL-4, IL-10, IL-13 or TGF $\beta$ stimulation, but not IFN $\gamma$, induced Arg 1 expression in murine macrophages. ${ }^{44,45}$ In line with previous findings, ${ }^{45}$ Arg 1 
a
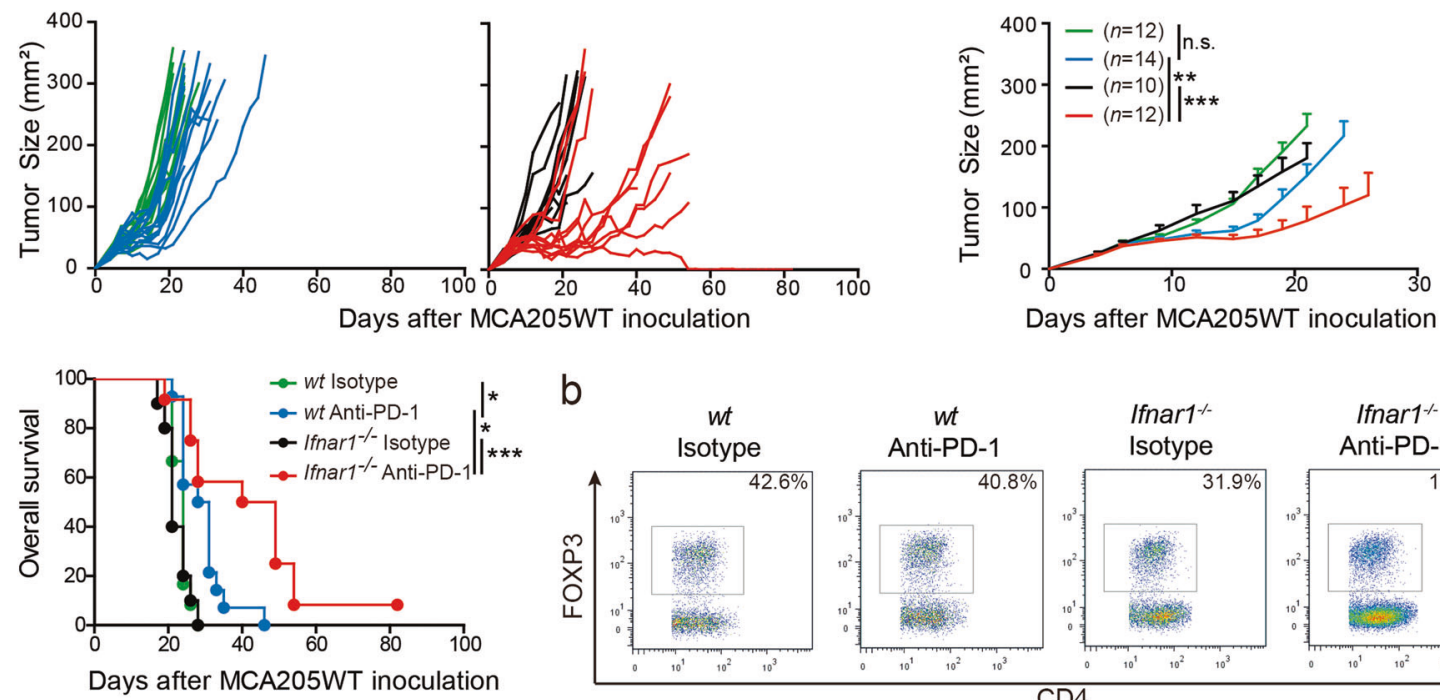

Days after MCA205WT inoculation
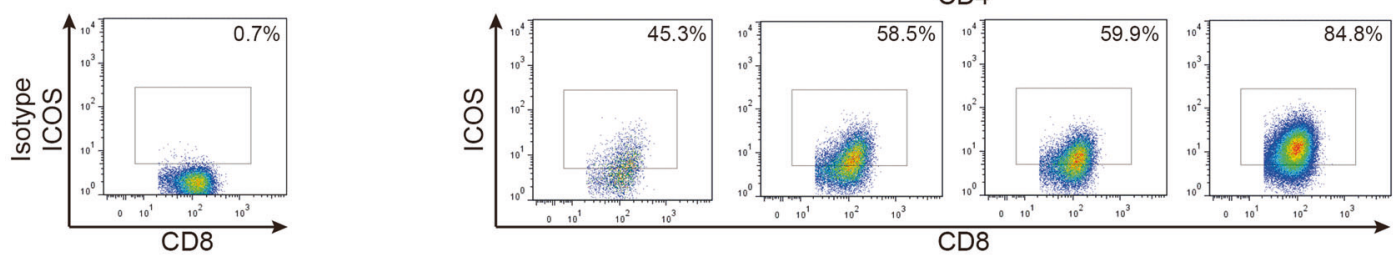

C

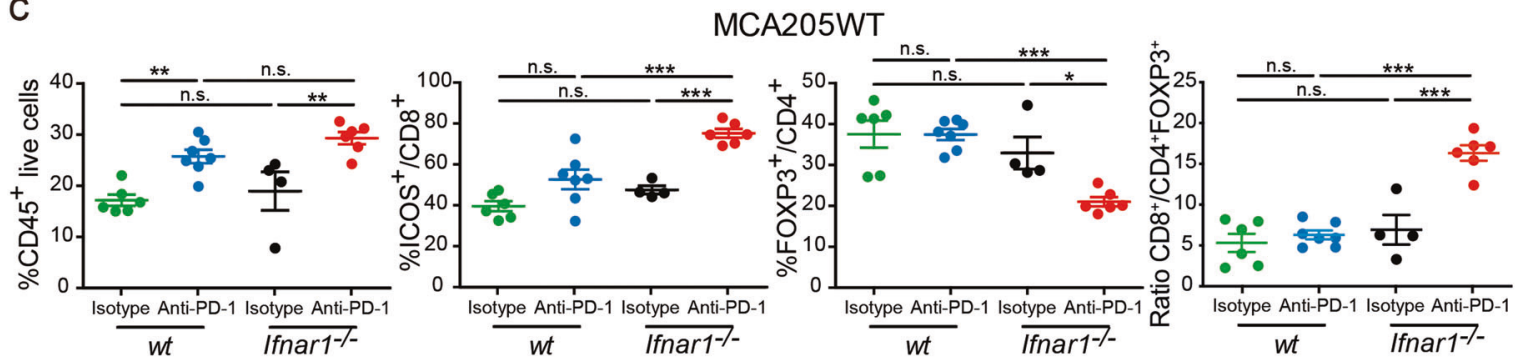

d
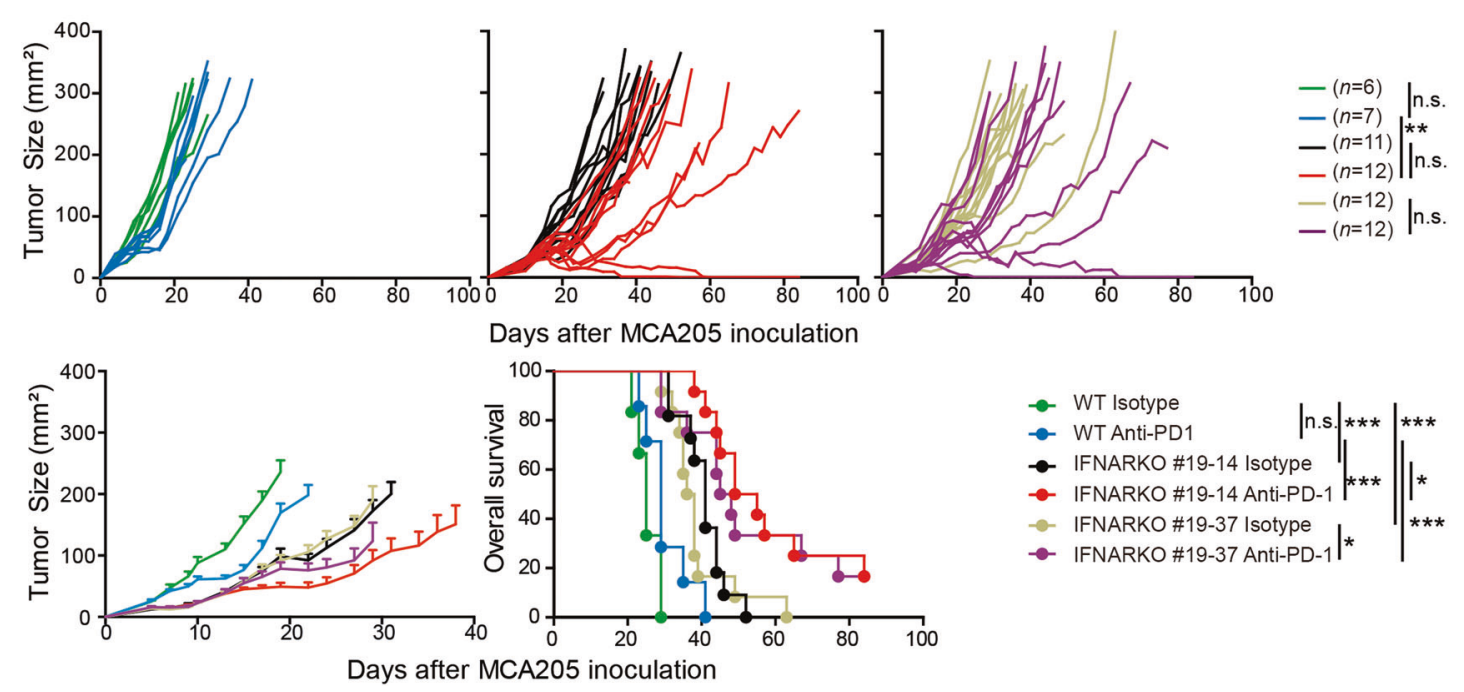

expression was not directly modulated by IFNs in BMDCs or BMMCs, or in tumor cells (Supplementary information, Fig. S7, Fig. S9a, b). Moreover, its in vivo expression was not modified by antiPD-1 therapy in either $\mathrm{CD} 45^{+}$or $\mathrm{CD} 45^{-}$fractions (Supplementary information, Fig. S9c, d). Finally, these results were corroborated using mice deficient in Arginase 1 expression in Tie2 positive cells demonstrating that the anti-tumor efficacy of the PD-1 blockade was not improved when arginase 1 was absent. (Supplementary information, Fig S9d).

Taken together, these findings indicate that Nos2 expression precedes the resistance to anti-PD-1 blockade. Furthermore, PD-1 therapy further increases NOS2 expression which is regulated by 
Fig. 2 Host and tumor IFNAR1 involved in secondary resistance to PD-1 blockade. a MCA205WT growth kinetics (top panels) and Kaplan-Meier survival curves (bottom panel) of WT and Ifnar $1^{-1-}$ C57BL/6 mice treated with anti-PD-1 mAb (or its isotype control) as described in Fig. 1a. $\mathbf{b}$ and $\mathbf{c}$ Representative gating strategy $\mathbf{b}$ and flow cytometry analyses $\mathbf{c}$ of tumor-infiltrating leukocytes after 4 injections and the proportions of CD45 ${ }^{+}$(left panel), ICOS ${ }^{+}$cells in the $\mathrm{CD} 8^{+} \mathrm{T}$ cell gate and $\mathrm{FOXP3}^{+}$cells in the CD4 ${ }^{+} \mathrm{T}$ cell gate (middle panels) and the ratio $\mathrm{CD}^{+} / \mathrm{CD}^{+} \mathrm{FOXP}^{+}$(right panel) are depicted. d Tumor growth kinetics and Kaplan-Meier survival curves of WT mice inoculated with two different clones of Ifnar $1^{-/-}$MCA205. Each line or dot represents one animal. The graphs represent 1 experiment (c) or depict pooled data from 2 a and $\mathbf{d}$ independent experiments encompassing 4-7 mice/group. For tumor growth and Kaplan-Meier curves, statistical analyses were performed using the specific software detailed in the Material and Methods. ANOVA statistical tests and pairwise comparisons with Bonferroni adjustment were used in $\mathbf{c}^{*}{ }^{*} p<0.05,{ }^{*} p<0.01,{ }^{* * *} p<0.001$, n.s.: not significant. Means \pm SEM are represented

type I (and type II) IFNs in both tumor cells and the tumor infiltrating myeloid fractions.

Critical role of the IFNAR/NOS2 signaling pathway in secondary resistance to PD-1 blockade

We next assessed the biological relevance of NOS2 function in adaptive resistance to PD-1 blockade. For this purpose, we treated MC38 colon cancers and MCA205WT sarcoma exhibiting early versus late resistance to anti-PD-1 mAb with the non-selective nitric oxide synthase inhibitor, N $\omega$-Nitro-L-arginine methyl ester hydrochloride (L-NAME). In sensitive MCA205WT sarcoma, L-NAME induced a sustained efficacy of anti-PD-1 mAb, beyond day 40, associated with long-term survival (Fig. 6a). To a lesser extent, LNAME also improved anti-PD-1 efficacy in MC38-bearing mice (Fig. 6b). Flow cytometric analyses of TILs revealed that L-NAME combined with an anti-PD-1 blockade tended to decrease tumorinfiltrating $\mathrm{CD}^{+}{ }^{+} \mathrm{FOXP3}{ }^{+}$regulatory $\mathrm{T}$ cells, and significantly increased the $\mathrm{CD}^{+} / \mathrm{CD}^{+} \mathrm{FOXP3}^{+}$ratio in MCA205WT tumors (Fig. 6c).

Additionally, we took advantage of Nos2-deficient mice which we inoculated with MCA205WT tumor cells. This experimental set up revealed the critical role of NOS2-expressing host cells in the resistance to the anti-PD-1 blockade. In fact, we did not observe any amelioration of the antitumor efficacy of PD-1 blockade in combination with L-NAME in Nos2-deficient mice whereas this combination markedly increased the anti-PD-1 treatment efficiency in WT mice (Fig. 6d).

Altogether, IFNAR signaling in leukocytes led to deleterious Nos2 expression contributing to progressive resistance to PD-1 blockade.

Clinical relevance of the type I IFN/NOS2 pathway in patients treated with anti-PD-1 mAb

We then analyzed the regulation of NOS2 gene expression by type I IFN in peripheral blood mononuclear cells (PBMC) from eight healthy volunteers (HV) and four melanoma (MEL) patients (Supplementary information, Fig. S10a, upper panel) together with TILs from seven stage III/IV MEL patients (Supplementary information, Fig. S10a, lower panel). As observed in mouse cells, we detected a significant upregulation of NOS2 (but not NOS3) and PDL1 gene expression in PBMCs but not in TILs at 24-60 h post-stimulation with type I IFN (Supplementary information, Fig. S10a).

We next examined NOS2 expression in tumor tissue in seven MEL patients treated with PD-1 blockade (patient characteristics detailed in Table S2). We carried out immuno-staining with an anti-NOS2 antibody upon paired biopsies, the first biopsy collected before treatment and the second biopsy collected during therapy or at relapse (Supplementary information, Fig. $\mathrm{S} 10 \mathrm{~b})$. We observed a notable increase in NOS2 expression during late resistance to anti-PD-1 mAb in three out of seven cases (Supplementary information, Fig. S10c), all of which were also remarkable for having undetectable NOS2 immunostaining prior to therapy.

To extend these initial findings, we investigated the gene transcripts associated with the response and resistance to PD-1 blockade immunotherapy with combined CTLA-4 co-blockade. We used a custom Nanostring panel to profile the expression of cancer/immune genes (Supplementary information, Table S3) in available pre-treatment tumors from 23 patients receiving combination anti-CTLA-4/PD-1 blockade for advanced melanoma (Andrews et al, manuscript in preparation) in a regimen that is often prescribed in the context of resistance to anti-PD-1 mAb. Differentially-expressed transcripts between responders $(\mathrm{R} ; n=17)$ and non-responders (NR; $n=6)$ revealed higher expression of several interferon-related genes (IFNA1, IFNA2, IFNA6, IFNA7, IFNA10, IFNA14) in NR tumors, consistent with our preclinical findings implicating type I IFN signaling in poor responses to immune checkpoint blockade (Fig. 7a). Also enriched in NR tumors were the inflammatory cytokine $I L 1 B$, and chemokine CXCL6 which has been implicated in melanoma growth and metastasis. ${ }^{46}$ There was no obvious correlation between receipt of prior immunotherapy and response or expression level of type I IFN or IFNAR1/2related genes, despite the majority of immunotherapy pre-treated patients $(n=5,71 \%)$ having received checkpoint blockade agents for the treatment of metastatic disease (Fig. 7b). However, nonresponding patients with a history of prior immunotherapy had numerically higher baseline levels of NOS2, but not IFNAR1/2, compared with immunotherapy pre-treated responders (Fig. 7c; Supplementary information, Fig. S11) suggesting a potential contribution of pre-activated NOS2 to treatment failure.

Therefore, the type I IFN pathway is activated in the TME at diagnosis or during treatment with immunomodulators eventually leading to Nos2 expression. This paves the way to resistance to cancer immunosurveillance.

\section{DISCUSSION}

By studying various tumor models exhibiting phenotypic traits of reduced, early or late resistance to a therapeutic antibody inhibiting the PD-1 immune checkpoint, we have unraveled IFNAR-induced NOS2 expression as a critical negative regulator of sustained anti-cancer efficacy of the PD-1 blockade that operates at the level of both tumor cells and leukocytes.

Mechanisms of resistance to proficient adaptive immune responses are being progressively dissected. Minn's group reported that both type I and II IFN maintain the resistance program to ICB-induced adaptive immunity in a PD-L1independent fashion. ${ }^{47}$ Indeed, both type I and II IFN signaling allowed tumors to acquire STAT1-related epigenomic changes, promoting the coordinated expression of IFN-stimulated genes and ligands for multiple $T$ cell inhibitory receptors. Knockdown of both IFN receptors on tumor cells considerably improved the response to the combination of radiotherapy with anti-CTLA-4 $\mathrm{mAb}$ through specific down regulation of genes associated with acquired resistance. Therefore, PD- $1^{\text {high }}$ Eomes ${ }^{\text {high }} \mathrm{T}$ cells expressing the whole panel of inhibitory receptors became exhausted upon engagement with IFN-induced ligands harbored by tumor cells, unless interventions on the tumor cell bottlenecks JAK1/JAK2 down-regulated the cascade culminating in surface expression of inhibitory ligands on tumor cells. ${ }^{47}$ Ifit and $M x 1$, when considered as a two-feature metagene, together with non-synonymous single nucleotide variant load, were the most relevant genomic features associated with resistance to PD-1 blockade in 27 melanoma 
a
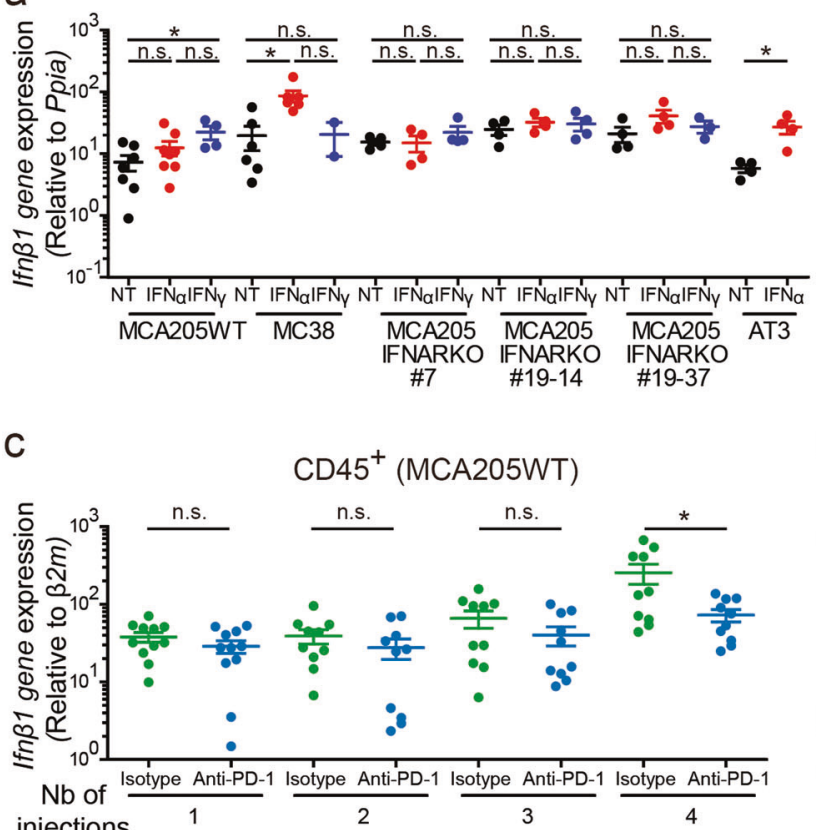

f

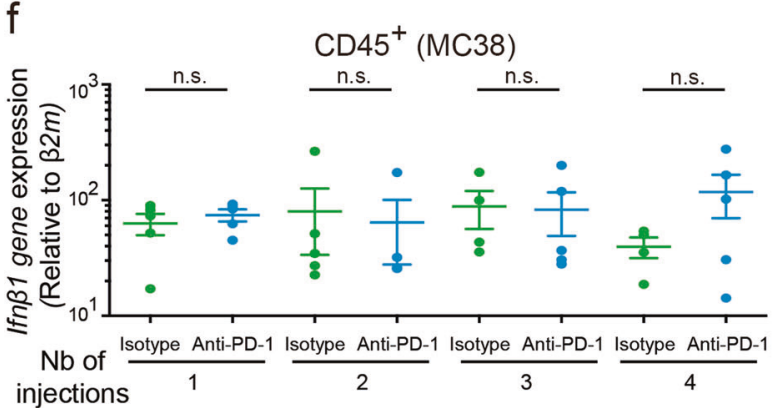

h

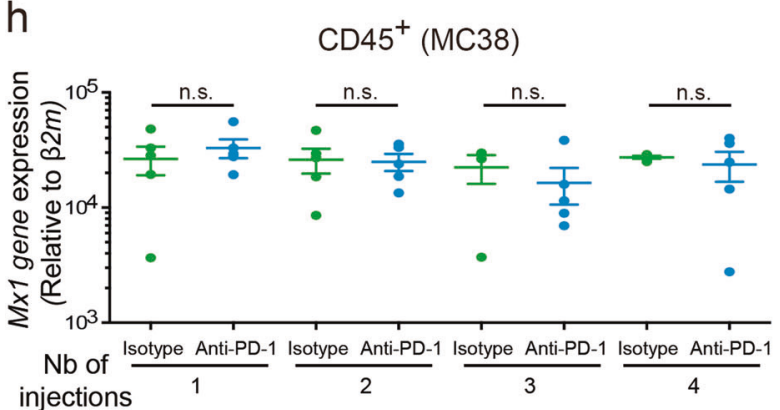

b

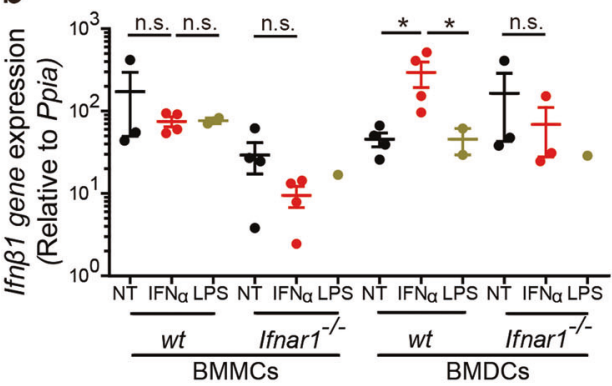

d

$\mathrm{CD}^{+} 5^{+}$(MCA205WT)

e
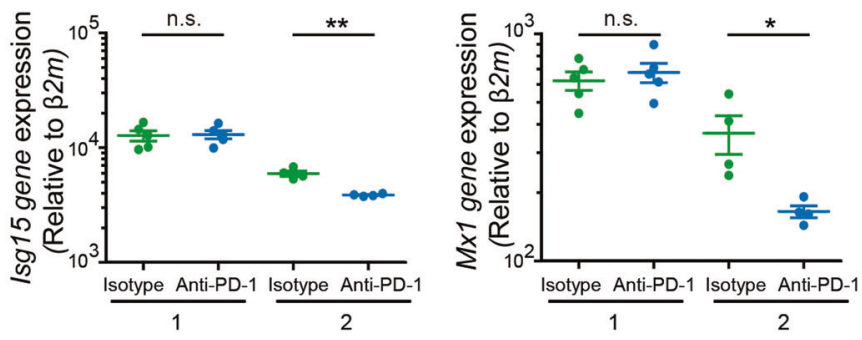

g

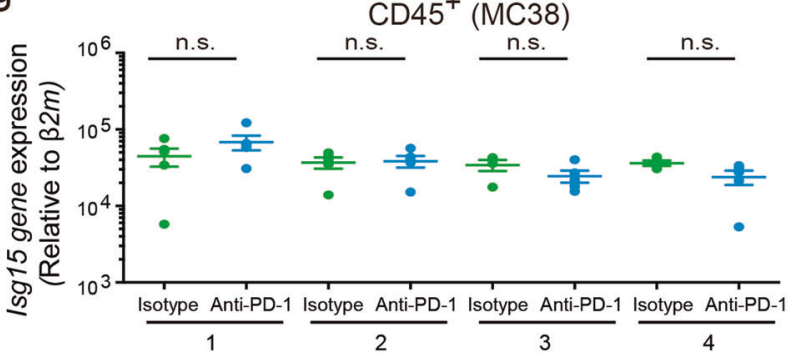

Fig. 3 Sources and kinetics of Type I IFN in the TME during PD-1 inhibition. $\mathbf{a}$ and $\mathbf{b}$ In vitro assays. Relative expression of Ifn $\beta 1$ quantified by qRT-PCR following stimulations of various tumor cell lines or BMDCs and BMMCs with IFN $\alpha$, IFN $\gamma$ or LPS. Each dot represents one sample and graphs represent 1 experiment or are the pool of 2 to 3 independent experiments including biological replicates for each experiment. Unpaired t-tests were used to compare 2 groups. ANOVA statistical tests and pairwise comparisons with Bonferroni adjustment were adopted for more than 2 groups. $\mathbf{c}-\mathbf{h}$ In vivo studies. Flow cytometry sorting of CD45 ${ }^{+}$live fractions from the TME of MCA205WT $\mathbf{c}-\mathbf{e}$ or MC38 $\mathbf{f}-\mathbf{h}$ tumors $48 \mathrm{~h}$ after 1, 2, 3 or 4 i.p. administrations of anti-PD-1 (or isotype control) mAb. Relative expression of Ifn $\beta 1$ c and $\mathbf{f}$ and IFN-sensitive gene products $\mathbf{d}, \mathbf{e}, \mathbf{g}, \mathbf{h}$ quantified by qRT-PCR. Unpaired t-tests were used to compared transcription levels between the anti-PD-1 and isotype control treated groups for each time point. Each dot represents 1 mouse with 5 mice per time point per experiment. Graphs represent 1 representative experiment out of 2-3 independent experiments (MC38, time points 1 and 2, d, e), 1 experiment (MC38, time points 3 and 4) or are the pool of $2-3$ independent experiments $c .{ }^{*} p<0.05,{ }^{* *} p<0.01$, n.s.: not significant. Mean \pm SEM are represented

patients. Hence, our preclinical study and our study of a clinical cohort of melanoma patients confirms the crucial role of cell autonomous type I IFN receptor signaling, IFNAR1, in the progressive loss of sustained efficacy of PD-1 blockade, and resistance to combination ICB, revealing a new mechanism for this puzzling resistance program.
Admittedly, such findings are at odds with previous reports showing that early production of type I IFNs promotes DC activation and $T$ cell cross-priming and that IFNAR signaling on host and tumor cells is crucial early during the effector phase, to ensure optimal antigen processing and minimal MHC-class I expression. In addition, two case reports described melanoma 
a

Day 0

tumor inoculation Anti-PD1 or Isotype control

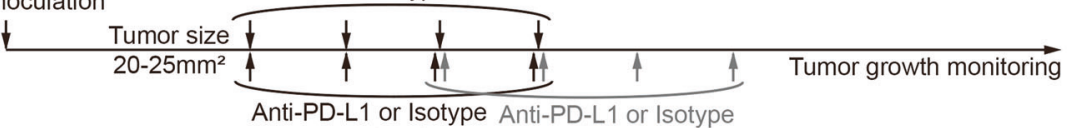

Anti-PD-L1 or Isotype Anti-PD-L1 or Isotype
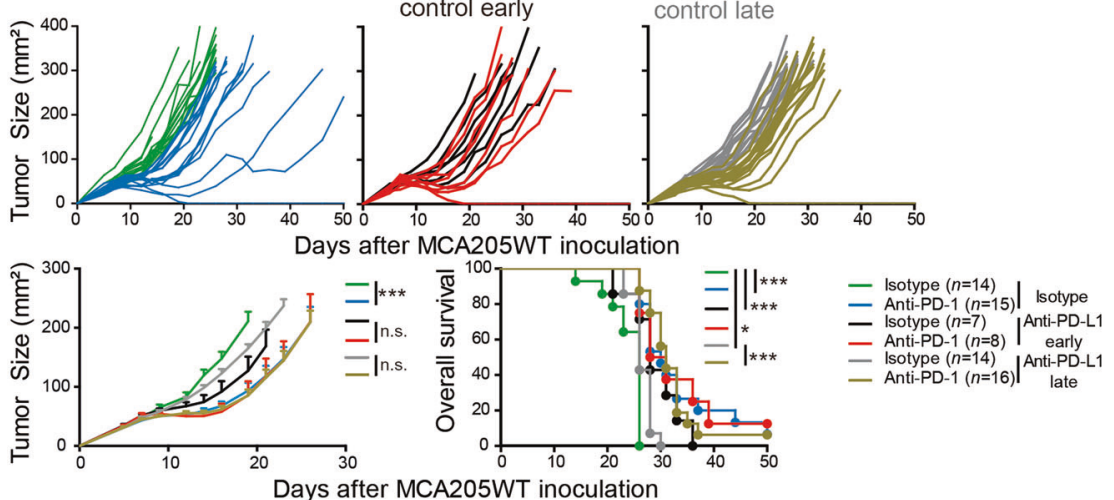

b
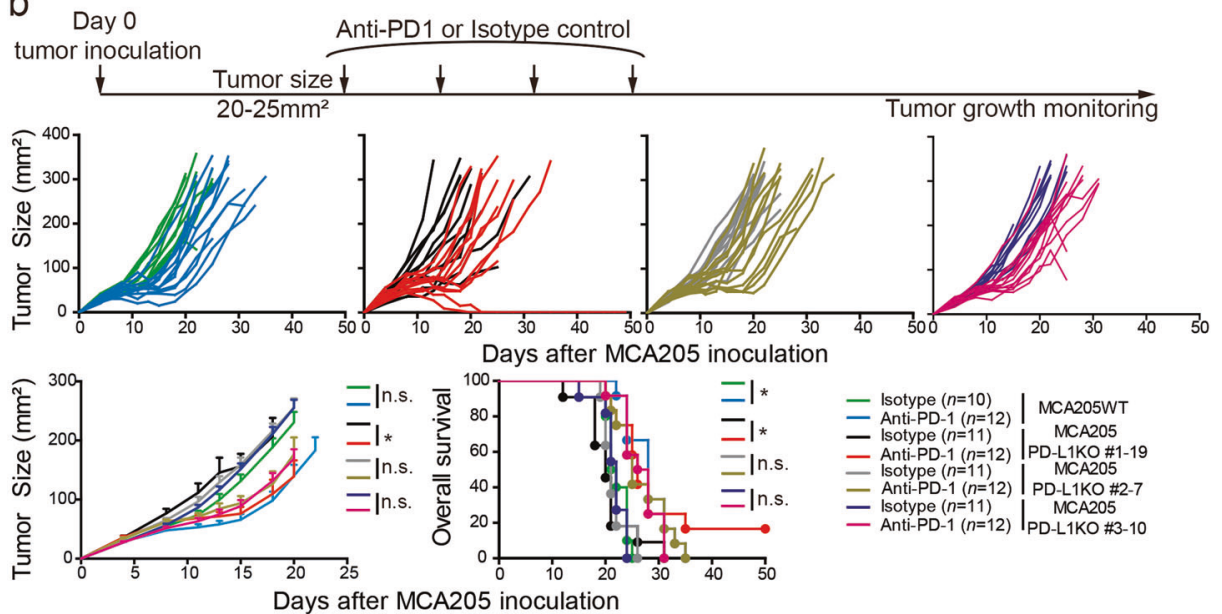

C Day 0

tumor inoculation Anti-PD1 or Isotype control
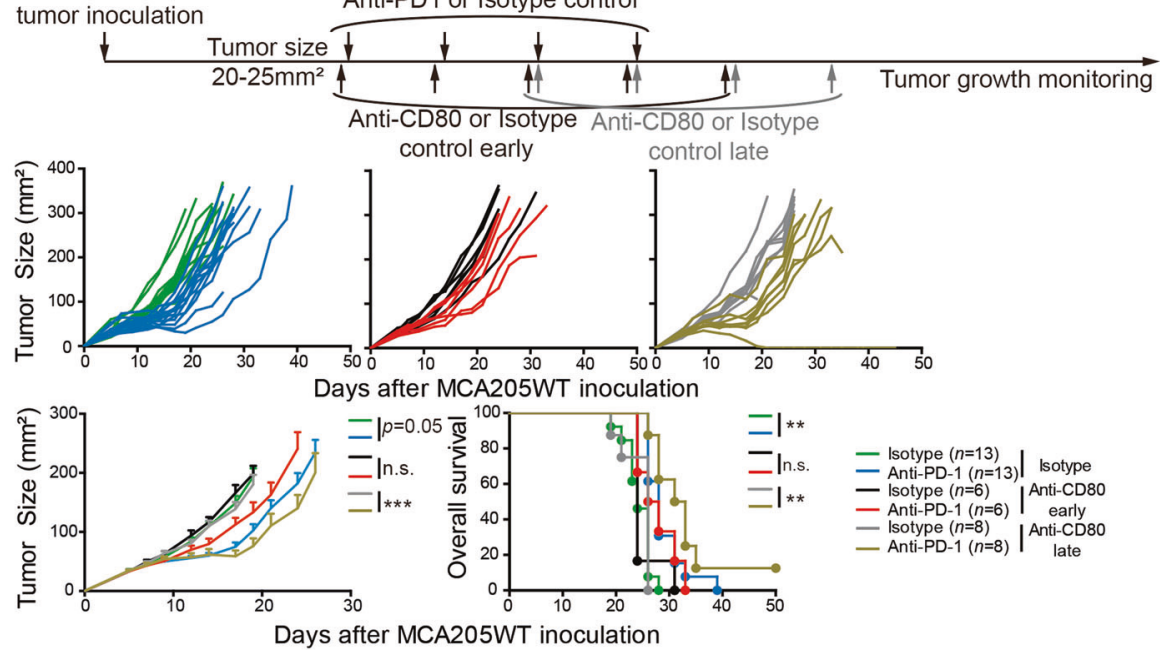

Fig. 4 Secondary resistance to PD-1 blockade is PD-L1 independent. a Effects of neutralizing anti-PD-L1 mAb co-administered early or late following initiation of anti-PD-1 mAb in MCA205WT tumor bearers. b Effects of anti-PD-1 mAb treatment on tumor growth against MCA205WT versus two different clones of $P d-11^{-1-}$ MCA205. c Equivalent schema as in a using anti-CD80 mAb. Each line or dot represents 1 animal. Mean \pm SEM are represented. Tumor growth kinetics and Kaplan-Meier survival curves are shown. The graphs depict tumor growth kinetics of 1 (a, anti-PD-L1 early; c, anti-CD80 early and late) or 2 independent experiments encompassing 5-8 mice/group and per experiment. Statistical analyses were performed using the specific software detailed in the Material and Methods. ${ }^{*} p<0.05,{ }^{* *} p<0.01,{ }^{* * *} p<$ 0.001 , n.s.: not significant 


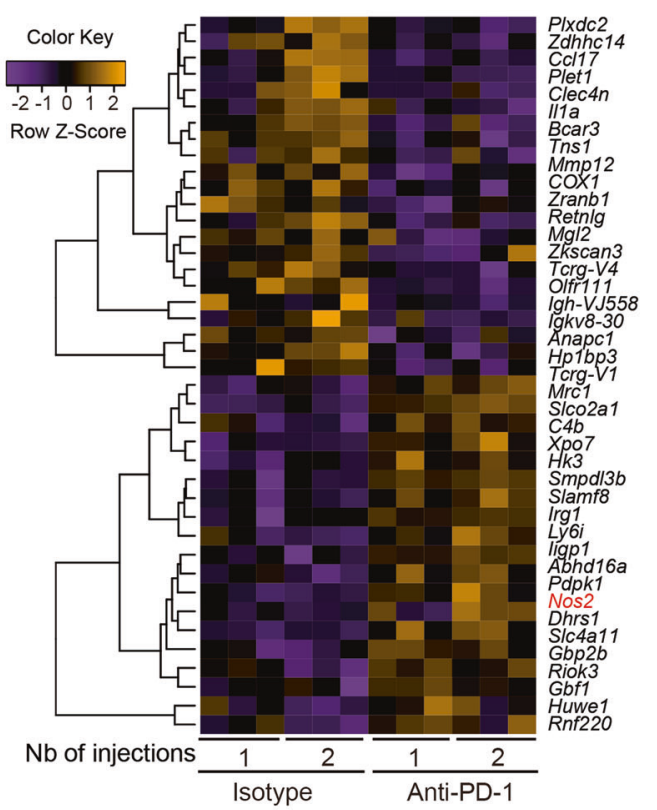

b
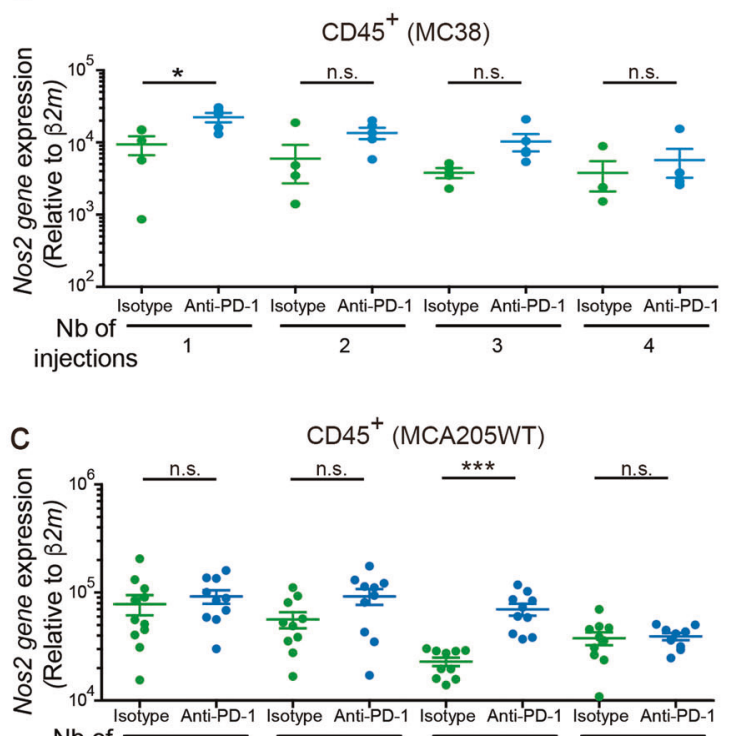

$\mathrm{Nb}$ of $\frac{1}{1} \frac{1}{2} \frac{1}{3} \frac{\text { Inpe Anti-PD-1 Isotype Anti-PD-1 Isotype Anti-PD-1 }}{4}$

d
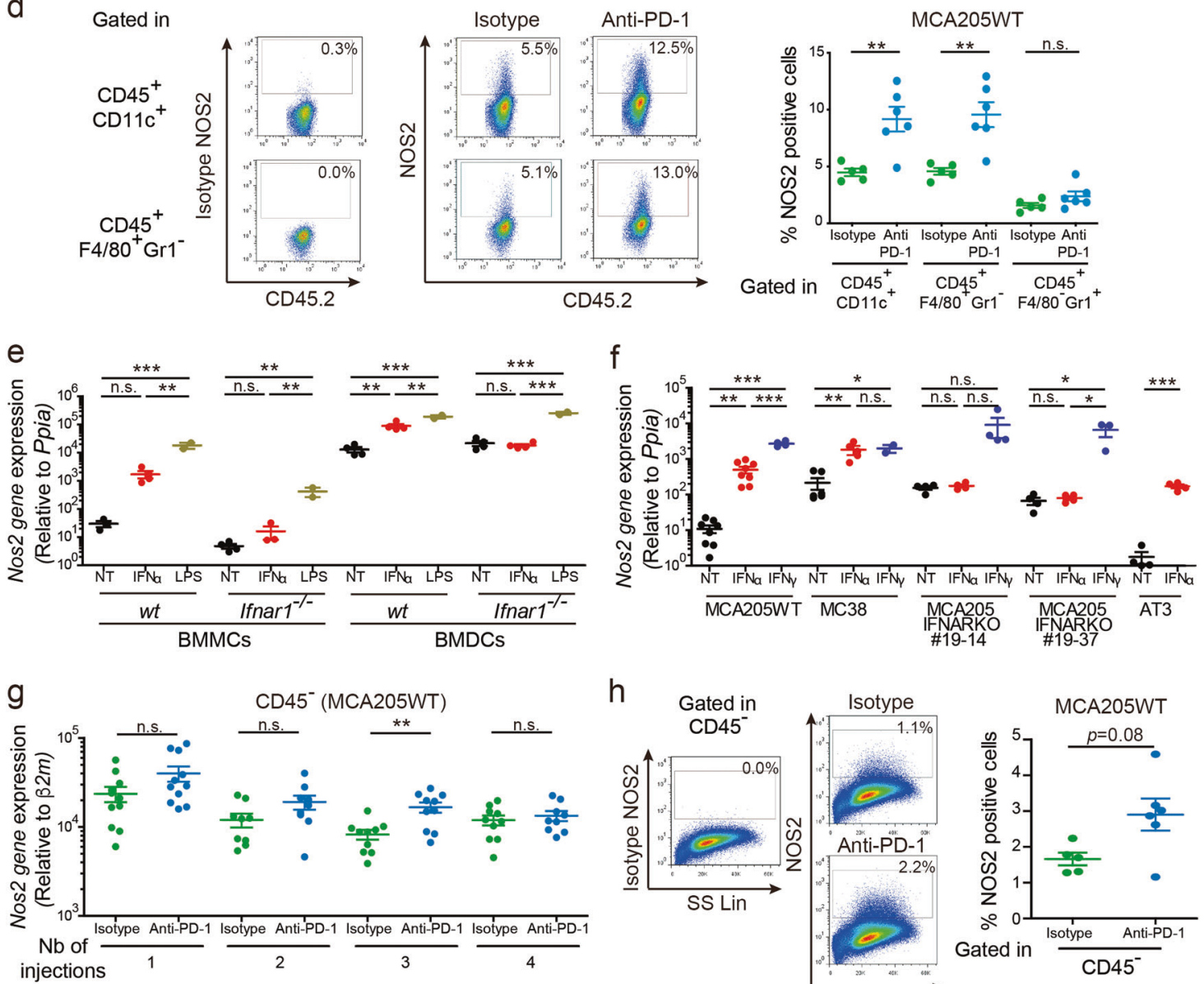

h
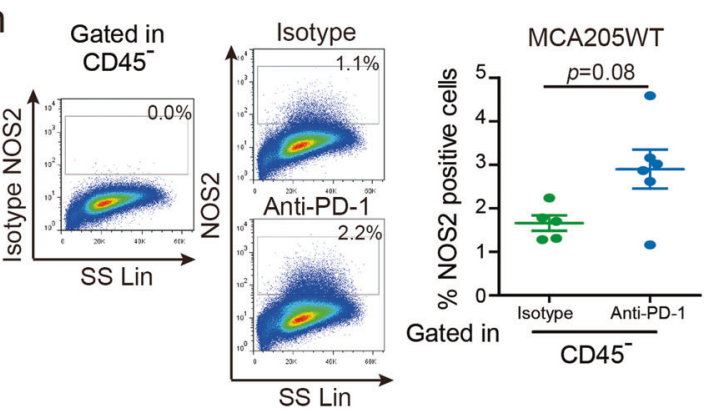

patients who initially responded to anti-PD-1 mAb but exhibited a late relapse attributed to loss-of-function mutations in JAK1 or $J A K 2{ }^{26}$ In parallel, another team reported melanoma patients who failed to respond to anti-CTLA-4 mAb whose tumors harbored copy number alterations in IFNY pathway genes. ${ }^{23}$ Together these

data suggest an emerging framework for resistance to ICB that consists of primary resistance, a Darwinian process culminating in the emergence of distinct tumor clones with selective and intrinsic growth/survival advantage (i.e.; "cold" tumors, MHC deficiencies, lack of relevant tumor antigens, PD-L1 expression without TILs, 
Fig. 5 Type I IFN-induced Nos2 expression post- PD-1 blockade in the TME. a Microarray analysis and qRT-PCR analyses of TILs in MC38 during PD-1 blockade. CD45 ${ }^{+}$cells from MC38 tumors were cell-sorted $48 \mathrm{~h}$ post-1, 2 injections of anti-PD- 1 or isotype control mAbs to perform trancriptomic analyses. Heat-map depicting the shared significant downregulated and upregulated genes in the anti-PD-1 treated groups compared with the isotype control treated groups across time points 1 and 2. b Relative expression of Nos2 in CD45 ${ }^{+}$cells from MC38 tumors $48 \mathrm{~h}$ after 1, 2, 3 and 4 injections of anti-PD-1 or Isotype mAbs using qRT-PCR analyses. $\mathbf{c}$ and $\mathbf{g}$ Same as b with MCA205WT tumor bearers after treatment with 1, 2, 3 and 4 injections of mAbs evaluated in both CD45 ${ }^{+} \mathbf{c}$ and $C D 45^{-} \mathbf{g}$ fractions. $\mathbf{d}$ and $\mathbf{h}$ Representative gating strategy and flow cytometric analyses of NOS2 protein expression in CD45 cells $\mathbf{d}$ and in the CD45 fraction $\mathbf{h}, 48 \mathrm{~h}$ after the fourth injection of anti-PD-1 or its isotype control mAbs. e, $\mathbf{f}$ Relative expression of Nos2 quantified by qRT-PCR following stimulations of BMDCs and BMMCs e or various tumor cell lines $\mathbf{f}$ with either IFN $\alpha$, IFN $\gamma$ or LPS. Each dot corresponds to one stimulated sample or 1 mouse with 2 or more biological replicates per experiment and 5 mice per group per time point per experiment. Graphs depict 1 experiment (b, time points 3 and 4 , $\mathbf{d}$ and $\mathbf{h}$ ), are representative of 1 experiment out of 2-3 performed (b, time points 1 and 2 ), or are the pool of 2-3 independent experiments $\mathbf{c}$, e-g including biological replicates for each experiment. Unpaired $t$-tests were used to compare two groups (b-d, $\mathbf{f}$ for AT3 tumor model and $\mathbf{g}$, $\mathbf{h}$ ). ANOVA statistical tests and pairwise comparisons with Bonferroni adjustment were adopted for more than 2 groups e, f. ${ }^{*} p<0.05$, ${ }^{* *} p<0.01$, ${ }^{* * *} p<$ 0.001 , n.s.: not significant. Means \pm SEM are represented

\begin{abstract}
"TGF $\beta$-like" transcriptional signature with concomitant upregulation of the expression of genes involved in mesenchymal transition, cell adhesion, extracellular matrix remodeling, angiogenesis, and wound healing), ${ }^{48}$ or acquired resistance, independent from PD-L1 expression, as a result of direct effector T cellmediated selective pressure. Both may be related to mutational or genetic events. The copy number loss in IFN pathway genes (such as Ifngr1, Irf1, Jak2, and Ifngr2), or the amplification of crucial IFNY pathway inhibitors, including SOCS1 and PIAS4 and loss-offunction mutations in JAK1/JAK2 represent paradigms of primary and/or acquired resistance mechanisms to ICB. From a teleological point of view, such genetic events may be selected to circumvent the cytostatic and/or cytotoxic effects of IFNs. ${ }^{23,26}$ However, in contrast to loss-of-function resistance mechanisms, adaptive resistance could be viewed as a negative feedback loop dulling anti-tumor $\mathrm{T}$ cell activity through functional IFN receptor signaling pathways, resulting in the upregulation of immunosuppressive PD-L1 or galectins ${ }^{47}$ or NOS2 as exemplified here. Interestingly, these theories extend not only to ICB or immune-based therapies but also to targeted therapeutic strategies. Genomic and transcriptomic features modulating the response to MAPK inhibitors (MAPKi) are being described in melanoma to account for the primary or secondary resistance. The genetic variants positively selected by MAPKi were not highly recurrent and could not fully explain clinical relapse. ${ }^{49}$ Instead, gene signature-based transcriptomic alterations in acquired MAPKi-resistant melanoma were highly recurrent, encompassing not only cell autonomous pathways (c-MET, LEF1, YAP1) but also patterns of $\mathrm{CD}^{+} \mathrm{T}$-cell exhaustion such as down-regulation of antigen presentation machinery, dominance of M2 macrophages and NF-kB activation in the TME. ${ }^{48,49}$
\end{abstract}

The production of nitric oxide (NO) in cells results from the conversion of L-arginine to L-citrulline by the NOS enzymes. NO regulates neurotransmission, immune responses and antimicrobial responses. Its role during the various stages of oncogenesis has also been well exemplified. NO acts at cell autonomous levels such as DNA damage, oncogene activation, inhibition of DNA repair enzymes, and tumor suppressor genes, modulation of apoptosis and metastases. ${ }^{50}$ The anti-tumor effects of NO produced by the immune defense were exemplified in various human tumors, while the pro-tumorigenic and immunosuppressive effects of NO (produced by M2 macrophages, MDSC, tumor or endothelial cells and neutrophils) were demonstrated in progressing tumors and metastases. $^{50}$ Therapy-induced NO can also translocate and increase aggressiveness of non-targeted bystander cells. ${ }^{51}$ NO mediates the nitration of tyrosine residues in multiple proteins, thereby lessening the Th1 gene signature in M1 macrophages. ${ }^{52}$

In our study, NOS2 was 100 to 1000 times more highly expressed than other NOS isoforms in the hematopoietic and cancer cell compartments. Therefore, the major source of NO in the tumors is due to the enzymatic activity of NOS2. The regulation of its expression in tumors has been previously widely studied. At the genetic and epigenetic level, polymorphisms in the Nos2 gene or DNA methylation in the Nos2 promotor influence activation or silencing of its enzymatic activity in tissues. ${ }^{50}$ Regarding non-genetic regulatory mechanisms, Nos2 is primarily regulated at the expression level by inflammatory cytokines (TNFa, IL-1 $\beta$, IL- 6 and IFN $)$, lipopolysaccharide, hypoxia, oxidative stress and HSP7O (ref. ${ }^{50}$ ). One report showed that Nos2 can be dependent on type I IFN signaling, especially STAT1, STAT2, Irf3 and NF-KB, involving pattern recognition receptors. ${ }^{53}$ Hence, our observation that NOS2 can be regulated in the TME by type I IFNs is original and adds to the complexity of NOS2 regulation. Supporting this notion, type I IFN-induced NOS2 in macrophages reduced intracellular accumulation of Leishmania major ${ }^{5,55}$ while favoring infection with Mycobacterium tuberculosis. ${ }^{56}$ IFNy-induced NOS2 was also shown to reduce $\mathrm{CD}^{+}{ }^{+} \mathrm{T}$ cell expansion in vitro following $\mathrm{T}$ cell activation with anti-PD-L1 or PD-1 mAbs, and NOS2 inhibitors were able to restore $\mathrm{CD}^{+}{ }^{+} \mathrm{T}$ cell proliferation. ${ }^{57}$

Our results indicate that the overexpression of Nos 2 in the context of PD-1 inhibition may occur early during treatment and, importantly, may precede resistance to anti-PD-1 therapy. Both the tumor and leukocyte cell fractions upregulate Nos2, the latter being mostly represented by $C D 11 c^{+} I A I E{ }^{\text {hi }}$ cells, with DC being the most prominent producers in vitro. Given that L-NAME failed to improve the antitumor efficacy of the anti-PD-1 mAb in Nos2deficient mice, and that L-NAME had some activity against distinct clones of Ifnar1-deficient sarcomas, we postulate that the functionally relevant source of NO is likely antigen presenting cells. The main mode of action of L-NAME was its capacity to activate DC and to reduce Treg accumulation in tumor beds, thereby increasing the $\mathrm{CD} 8^{+} / \mathrm{FOXP} 3$ ratio in the context of a longterm PD-1 blockade. Therefore, our study supports the development of strategies aimed at restraining NOS2 activity and/or NO release to counteract PD-L1-independent resistance pathways elicited during PD-1 blockade.

\section{MATERIAL AND METHODS}

Mouse studies

All experiments were approved by the local institutional boards. Experiments were performed in accordance with government and institutional guidelines and regulations. Ifnar ${ }^{-1-}$ mice were originally kindly provided by the Professor Gilles Uzé (Université de Montpellier II, Montpellier, France) and the strain was maintained in the animal facility of Gustave Roussy Cancer Campus. Nos2 $2^{-1-}$ mice were kindly provided by either the Doctor Armelle Prevost-Blondel (Institut Cochin, Paris, France) or by the Professor Vincenzo Bronte (Verona University Hospital, Verona, Italy). Arg $1^{f / f l}$ Tie2 $^{\mathrm{Cre} /+}$ and $\operatorname{Arg} 1^{f / f l}$ mice were kindly provided by the Professor Vincenzo Bronte (Istituto Oncologico Veneto, Padova, Italy). ${ }^{58}$ Females and males C57BL/6 were purchased from Harlan (France). Only females were used in experiments with the exception of specific KO strains and their respective controls. 

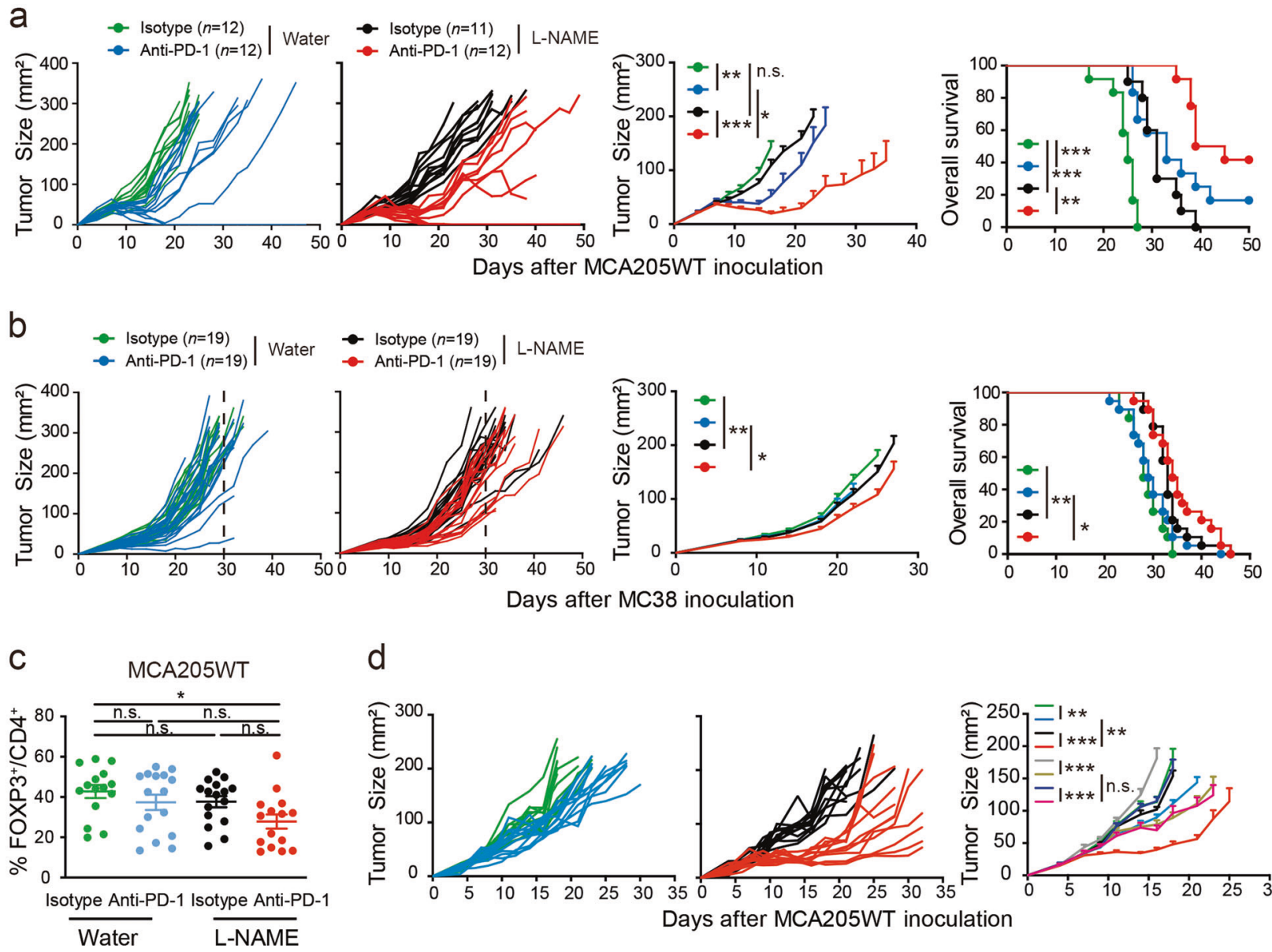

d
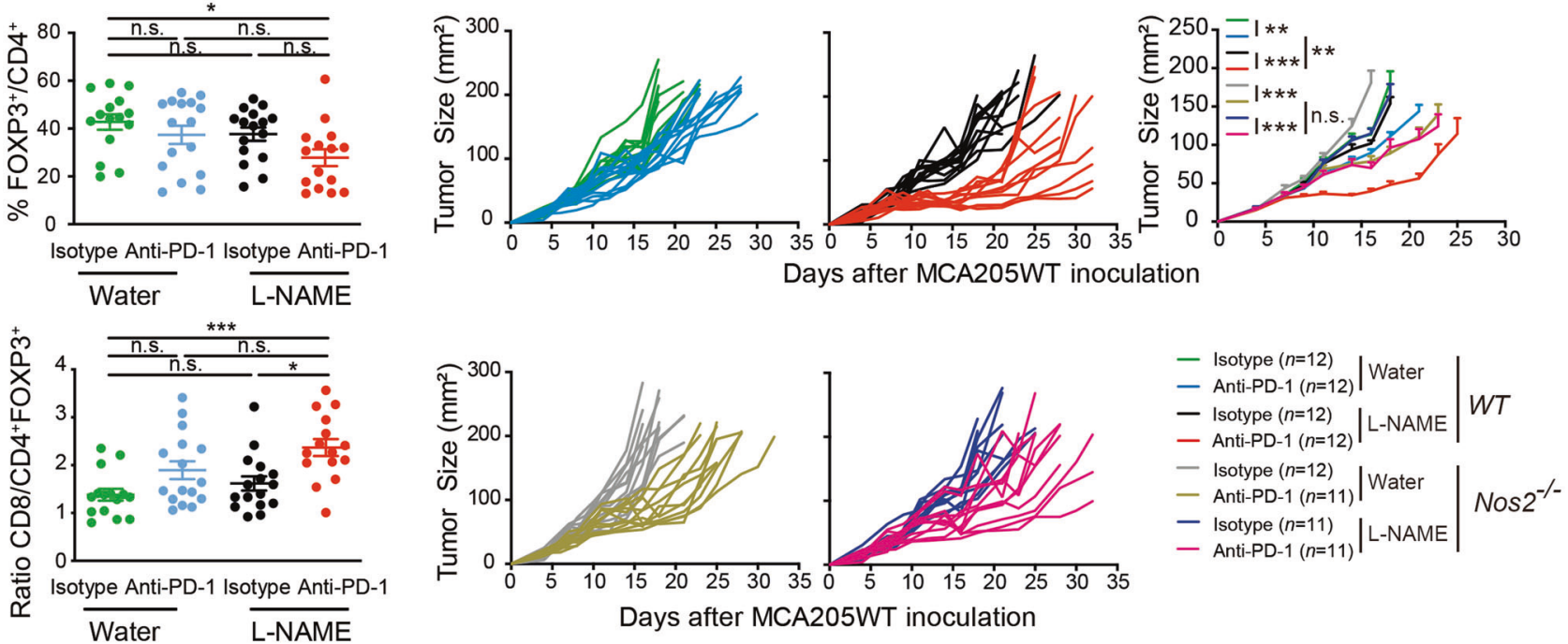

Fig. 6 Pharmacological inhibition of NOS2 ameliorated the efficacy of PD-1 blockade in various tumor models. Concomitant blockade and inhibition of PD-1 and NOS2 with L-NAME in established MCA205WT a and MC38 b tumors. Tumor growth kinetics (left and middle panels) and survival curves (right panels) are depicted for each group. L-NAME treatment (1 g/L) was started one day prior to anti-PD-1 infusion and was maintained until the end of the experiment. The graphs depict tumor growth kinetics of a pool of 2-3 independent experiments encompassing 5-9 mice per group and per experiment. c Flow cytometry analyses of TILs after 4 injections focusing on the proportion of FOXP3 $^{+}$cells among CD4 ${ }^{+}$T cells and the ratio of $\mathrm{CD}^{+}{ }^{+}$T cells/CD4 ${ }^{+} \mathrm{FOXP3}^{+}$Treg cells are depicted. Each dot represents 1 animal and graphs depict the pool of 3 independent experiments. Means \pm SEM are represented. Statistical analyses were performed using ANOVA statistical tests and pairwise comparisons with Bonferroni adjustment. d Effect of L-NAME in anti-PD-1-treated MCA205WT tumors inoculated into WT or Nos $2^{-/-}$mice. The graphs depict a pool of 2 independent experiments including 5-7 mice/group and per experiment. Statistical analyses were performed using the specific software detailed in the Material and Methods. ${ }^{*} p<0.05,{ }^{* *} p<0.01,{ }^{* * *} p<0.001$, n.s.: not significant

Mice were used between 8 and 16 weeks of age. All mice experiments were performed at Gustave Roussy Cancer Campus, France or at the Istituto Oncologico Veneto, Italy and mice were housed in specific pathogen-free conditions in both animal facilities or were maintained in isolators.

Mouse cell lines

The AT3 cell line was kindly provided by the Professor Mark Smyth (QIMR Berghofer Medical Research Institute, Brisbane, Australia). MCA205 derived IFNAR1 and PD-L1 KO clones were generated as described below. The MCA205WT and MCA2050VA sarcoma,
MC38 carcinoma and AT3 mammary carcinoma cell lines were cultured at $37^{\circ} \mathrm{C}$ under $5 \% \mathrm{CO}_{2}$ in RPMI-1640 medium supplemented with $10 \%$ heat-inactivated fetal bovine serum (FBS), $1 \%$ penicillin/streptomycin, $2 \mathrm{mM}$ L-glutamine and $1 \%$ of sodium pyruvate and non-essential amino acids (all from Gibco-Invitrogen), referred herein as complete RPMI medium. The right flank of mice was subcutaneously (s.c.) injected with $0.8 \times 10^{6}$ cells for MCA205WT and $1 \times 10^{6}$ cells for MCA205OVA, MC38 and AT3. Mouse cell lines were regularly tested for mycoplasma contamination and cells were not used for more than 10 passages. 
a

Differentially-expressed genes between $R$ and NR patients to CTLA-4 and PD-1 blockade in baseline tumor samples

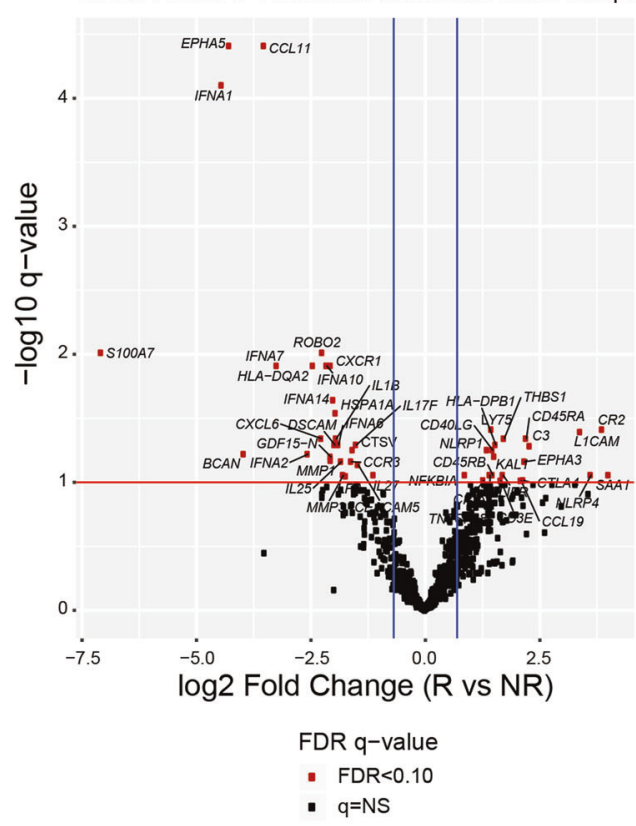

C

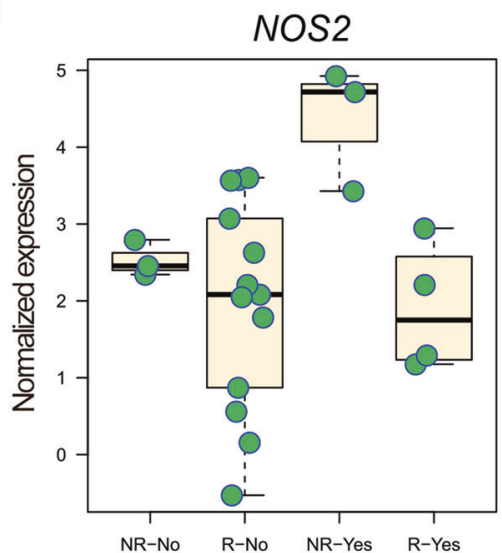

Response - Prior Immunotherapy b

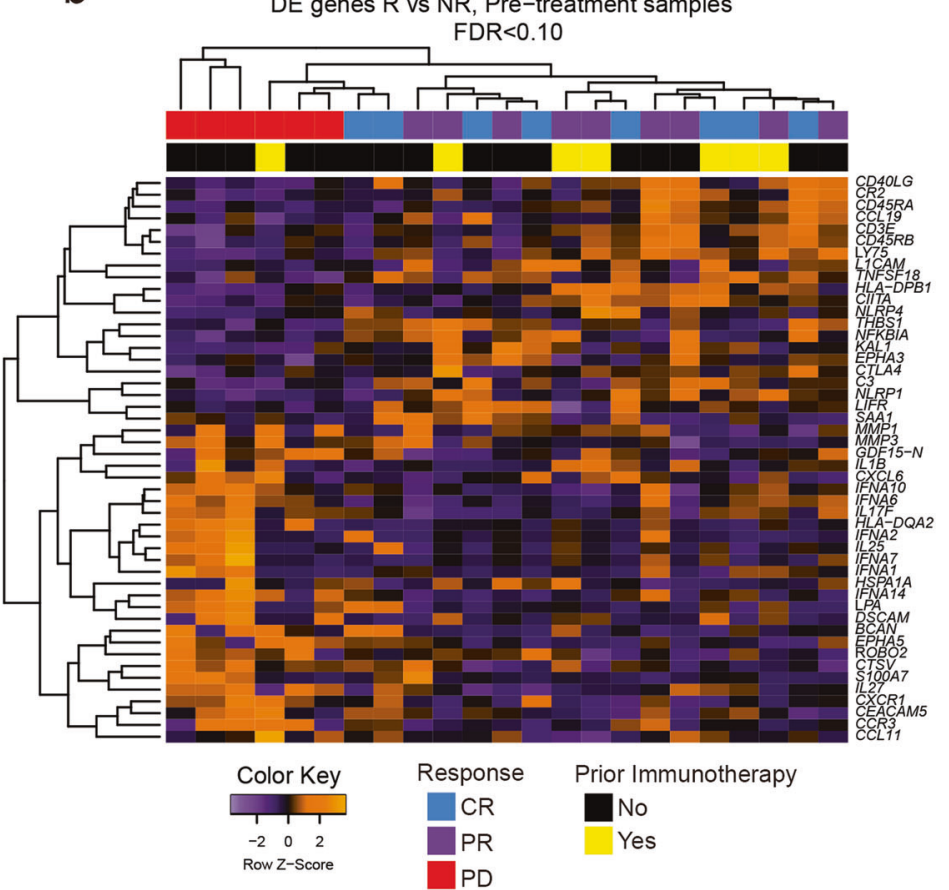

Fig. 7 Type I IFN and NOS2 are associated with the secondary resistance of anti-PD-1 + anti-CTLA-4 therapy in patients. a Volcano plot of NanoString gene expression analysis in tumor biopsies harvested prior to initiation of combination anti-CTLA-4 and anti-PD-1 immune checkpoint blockade comparing responders (R) versus non-responders (NR). b Heatmap of differentially-expressed genes (FDR $<0.10)$ in pretreatment samples comparing $R$ versus NR patients, indicating RECIST-based best overall response $(C R=$ complete response, $P R=$ partial response, $\mathrm{PD}=$ progressive disease), and receipt of prior melanoma-directed systemic immunotherapy (cytokine, checkpoint blockade agent). c Boxplots of NOS2 gene expression stratified by response to combination immune checkpoint blockade $(R=r e s p o n d e r, ~ N R=n o n-r e s p o n d e r)$ and prior immunotherapy status (Yes/No) demonstrating numerically higher NOS2 levels in NR patients with prior immunotherapy exposure

CRISPR/Cas9 and Zinc Finger clones

Ifnar $1^{-1-}$ MCA205 cell lines were generated by means of the CompoZr ${ }^{\oplus}$ Zinc Finger Nuclease Technology (Sigma-Aldrich), as per manufacturer's recommendations as previously reported. ${ }^{38,59}$ $\mathrm{Pd}_{11^{-1-}}$ MCA205 cell lines were generated using the CRISPR/Cas9 technology (pCMV-Cas9-GFP, Sigma-Aldrich), as per manufacturers' protocols. After transfection, single GFP positive cells were sorted in 96 well-plates for the establishment of different clones. After the generation of clones, IFNAR1 and PD-L1 expressions were checked by flow cytometry and functional assays.

Bone marrow derived-DC and myeloid cell cultures

Bone marrow-derived dendritic cells (BMDCs) and bone marrowderived myeloid cells (BMMCs) were generated using femurs and tibias of females C57BL/6 WT and Ifnar $1^{-1-}$ mice aged of 8 to 12 weeks. Bones were carefully collected in sterile PBS. After washing bones in alcohol and Iscove's medium (IMDM, SigmaAldrich) baths, extremities of bones were cut and flushed using a $26 \mathrm{G}$ needle. After red blood cell lysis with ACK buffer, cells were cultured in IMDM supplemented with $10 \%$ of FCS $+2 \mathrm{mM}$ LGlutamine + $100 \mathrm{UI} / \mathrm{mL}$ Penicillin/Streptomycin + $50 \mu \mathrm{M}$ 2mercaptoethanol (Sigma-Aldrich) (referred herein as complete IMDM medium) at $0.5 \times 10^{6} / \mathrm{mL}$ and treated with $10 \mathrm{ng} / \mathrm{mL}$ of GM-CSF and IL-4 for BMDCs and $50 \mathrm{ng} / \mathrm{mL}$ of M-CSF for BMMCs (all from Peprotech). Cells were split at day 3 and used at day 7 or 8.

Therapies

In vitro stimulations. Tumor cell lines were cultured in complete RPMI medium at $0.5 \times 10^{6} / \mathrm{mL}$ in 48 well plates in triplicates and 
treated with IFNa (from Miltenyi Biotech) or IFNy (eBioscience) at $1000 \mathrm{IU} / \mathrm{mL}$. BMDCs or BMMCs cells were cultured in complete IMDM at $0.5 \times 10^{6} / \mathrm{mL}$ in 48 well-plates in triplicates and treated with $1000 \mathrm{IU} / \mathrm{mL}$ of IFNa (from Miltenyi Biotech, Germany) or $100 \mathrm{ng} / \mathrm{mL}$ of ultrapure LPS (InvivoGen). After $24 \mathrm{~h}$ of incubation, supernatants were collected and stored at $-80^{\circ} \mathrm{C}$ until cytokine measurements. In parallel, cells were harvested, washed and pre-incubated with anti-CD16/32 Ab (clone 93, eBioscience) for $20 \mathrm{~min}$ at $4{ }^{\circ} \mathrm{C}$ and then stained to discriminate populations and markers of interest using the antibodies listed in Supplementary information, Table S4. In vivo treatments. Mice were treated intraperitoneally (i.p.) when tumors became palpable (20 to $\left.25 \mathrm{~mm}^{2}\right)$ with anti-PD- $1 \mathrm{mAb}(250 \mu \mathrm{g} / \mathrm{mouse}$; clone RMP1-14, BioXcell, NH, USA), with or without anti-CD80 $(500 \mu \mathrm{g} / \mathrm{mouse}$; clone 1G10, BioXcell, NH, USA), anti-PD-L1 (250 $\mu \mathrm{g} /$ mouse; clone 10 F.9G2, BioXcell, NH, USA), anti-CTLA-4 (100 $\mu \mathrm{g} /$ mouse; clone 9D9, BioXcell, $\mathrm{NH}, \mathrm{USA}$ ) (or the appropriate isotype control) $\mathrm{mAb}$ as described in the figures or figure legends. Early or late injections correspond to one day prior (anti-CD80 early) or the same day (anti-PD-L1 early) as the first anti-PD-1 infusions or one day prior (anti-CD80 late) or concomitantly (anti-PD-L1 late) with the third anti-PD-1 treatment, respectively, for four injections every three days. Abatacept was kindly given by Professor Antoine Durrbach (Hôpital Bicêtre, Le Kremlin-Bicêtre, France) and dissolved in sterile PBS prior to i.p. injections in mice at $10 \mathrm{mg} / \mathrm{kg}$. Inhibition of NOS was performed using L-NAME dissolved in drinking water, given ad libitum, at $1 \mathrm{~g} / \mathrm{L}$ started one day prior anti-PD-1 $A b$-based treatment until the end of the experiment. Bottles were changed every 2 to 3 days.

\section{Flow cytometry analyses and cell-sorting}

Mice were sacrificed 2-3 days after the final anti-PD-1 or isotype control antibody treatments to assess immune parameters in the spleen and in the tumor bed. Briefly, tumors were cut into small pieces and digested in RPMI medium containing Liberase ${ }^{\text {TM }}$ at $25 \mu \mathrm{g} / \mathrm{mL}$ (Roche) and DNase 1 at $150 \mathrm{IU} / \mathrm{mL}$ (Roche) for $30 \mathrm{~min}$ at $37^{\circ} \mathrm{C}$. Cells were then filtered through a $100-\mu \mathrm{m}$ cell strainer, and splenocytes at a $2 \times 10^{6} / \mathrm{mL}$ concentration (after red blood cell lysis) or TME-derived cells were preincubated with purified antimouse CD16/32 mAb (clone 93, eBioscience) for $20 \mathrm{~min}$ at $4{ }^{\circ} \mathrm{C}$ before membrane staining. Dead cells were excluded using the live/dead fixable yellow dead cell stain kit (Life Technologies ${ }^{\mathrm{TM}}$ ). For membrane staining, antibodies were incubated $20 \mathrm{~min}$ at $4{ }^{\circ} \mathrm{C}$ to discriminate populations of interest. To assess intracellular cytokine production, $2 \times 10^{6}$ cells were stimulated with PMA (50 $\mathrm{ng} / \mathrm{mL}$ ) and ionomycine $(1 \mu \mathrm{g} / \mathrm{mL}$ ) (all from Sigma Aldrich) in the presence of Golgi Stop (BD Biosciences). After $4 \mathrm{~h}$ of stimulation at $37^{\circ} \mathrm{C}$, cells were membrane stained and then permeabilized using BD Cytofix/Cytoperm Kit (BD Biosciences) during 20 min at $4{ }^{\circ} \mathrm{C}$, washed and intracellularly stained during $30 \mathrm{~min}$ at $4{ }^{\circ} \mathrm{C}$ with antiTNFa, anti-IFN $y$ and anti-IL-17A Abs. For Nos2 and Arg1 protein level expressions, cells were membrane stained, permeabilized using BD Cytofix/Cytoperm Kit (BD Biosciences) during $20 \mathrm{~min}$ at $4{ }^{\circ} \mathrm{C}$, washed and then intracellularly stained during $30 \mathrm{~min}$ at $4{ }^{\circ} \mathrm{C}$ with anti-Nos2 and anti-Arg1 Abs. To assess cell proliferation and proportion of regulatory $\mathrm{CD} 4^{+} \mathrm{T}$ cells after surface staining, cells were permeabilized during $45 \mathrm{~min}$ at $4{ }^{\circ} \mathrm{C}$ using the Foxp3 kit (eBioscience) and then washed and stained with anti-Ki67 and anti-Foxp3 Abs during $30 \mathrm{~min}$ at $4{ }^{\circ} \mathrm{C}$. Antibodies are detailed in the Supplementary information, Table S4. Acquisition was performed on a Cyan ADP 9 Color Flow Cytometer (Beckman Coulter) after appropriate compensation using mono-stained cells. Data were analyzed with FlowJo software (Tree Star), version 7.6.5. To isolate cells following tumor digestion, the digested tissue was incubated in Fc-block and then stained during $20 \mathrm{~min}$ at $4{ }^{\circ} \mathrm{C}$ with anti-CD45.2 Ab in Automacs Buffer (Miltenyi Biotech). Cell sorting was carried out on either a BD Influx or a BD Aria III Flow cytometer. Cells were immediately resuspended in $\mathrm{RLT}^{+}$Buffer and stored at $-80^{\circ} \mathrm{C}$ until RNA extraction.

\section{Gene expression analyses}

Culture conditions. Tumor cell lines and BMMCs/BMDCs were cultured at $0.02 \times 10^{6} / \mathrm{mL}$ in 96 well flat bottom-plates (Nunc MaxiSorp, sterilized during $30 \mathrm{~min}$ under UV) in complete medium and treated either with IFNa (from Miltenyi Biotech), IFNy (eBioscience), both at $1000 \mathrm{IU} / \mathrm{mL}$ or $100 \mathrm{ng} / \mathrm{mL}$ of ultrapure LPS (InvivoGen). $24 \mathrm{~h}$ later, supernatant was collected after centrifugation and 12 wells were pooled together in $\mathrm{RLT}^{+}$buffer to form one replicate and kept for gene expression analyses. RNA extraction. Total RNA extraction and genomic DNA removal were performed with the RNeasy Mini kit (Qiagen), following the manufacturer's recommendations. Reverse transcription. A maximum of $1 \mu \mathrm{g}$ of RNA, measured by using a NanoDrop ${ }^{\text {TM }}$ Spectrophotometer (Thermo Fischer Scientific), was reverse transcribed into cDNA with a mix composed of SuperScript III Reverse Transcriptase (Life Technologies), RNaseOUT ${ }^{\mathrm{TM}}$ Recombinant Ribonuclease Inhibitor (Life Technologies), Random primers (Promega) and Deoxynucleoside Triphosphate Set, PCR grade (Roche Diagnostics). Quantitative gene expression assay. Expression of $\beta 2 \mathrm{~m}$ (Mm00437762_m1), Ppia (Mm02342429_g1), Ifnß1 (Mm00439552_s1), Nos2 (Mm00440502_m1), Nos1 (Mm01208059_m1), Nos3 (Mm00435217_m1), Arg1 (Mm004 75988_m1), Isg15 (Mm01705338_s1), Mx1 (Mm01218004_m1), and Pdl1 (Mm0045054_m1) (all from Life Technologies) was analyzed with the $\operatorname{TaqMan}^{\circledR}$ Gene Expression Assay using the Universal Master Mix II on a StepOnePlus ${ }^{\mathrm{TM}}$ Real-Time PCR System (Life Technologies). Amplifications were carried out using the following ramping profile: $1 \mathrm{cycle}$ at $95^{\circ} \mathrm{C}$ for $10 \mathrm{~min}$, followed by 45 cycles of $95^{\circ} \mathrm{C}$ for $30 \mathrm{~s}, 60^{\circ} \mathrm{C}$ for $1 \mathrm{~min}$. Quantitative RT-PCR data were normalized to the expression levels of the housekeeping genes $\beta 2 \mathrm{~m}$ or Ppia, as indicated in each figure, by means of the $2^{-\Delta \mathrm{Ct}}$ method multiplied by $10^{6}$.

\section{Cytokine quantification}

CXCL10 was measured by ELISA (BD Biosciences) according to the manufacturer's recommendations.

\section{Microarray analysis}

The $\mathrm{CD}_{4} 5^{+}$fraction from MC38 tumors was subjected to cellsorting and extracted as described above. RNA was subjected to control quality using a Bioanalyzer 2100. GeneChip Mouse Gene 2.0ST arrays (Affymetrix) were used to analyze the gene expression profile of $\mathrm{CD}_{4} 5^{+}$samples. This was performed at the Genomics Platform of the Cochin Institute according to standard validated protocols. Data analyses and representations were performed with the R software (http://www.R-project.org/). To assess the statistical significance of the differential gene expression, the empirical Bayes statistics for linear model (series of probeset arrays) was used (limma package). ${ }^{60}$ Selection criteria were as follows: |logFC| $>\log _{2}(1.5), p$-value $<0.05$. Heatmap representations are normalized by row. The raw data are available upon request to the lead author.

\section{Human studies}

All patients provided informed consent before enrollment in these studies. In vitro stimulations. Peripheral blood mononuclear cells (PBMCs) were isolated from blood of healthy volunteers using a Ficoll-Hypaque density gradient media (PAA Laboratories). After centrifugation, PBMCs were collected, washed, counted and then used for in vitro experiments. Tumor infiltrated leukocytes (TILs) were isolated from metastatic lesions of melanoma patients as previously described. ${ }^{61}$ Briefly, tumor samples were minced and then digested using a mix of Collagenase IV $(50 \mathrm{IU} / \mathrm{mL})$, hyaluronidase $(280 \mathrm{IU} / \mathrm{mL}$ ) and Dnase1 $(30 \mathrm{IU} / \mathrm{mL}$ ) (all from Sigma-Aldrich) in RPMI $1640+1 \%$ penicillin/streptomycin (Gibco 
Inviotrogen). Samples were subjected to gentleMACS dissociation (Miltenyi Biotech). Digested samples were washed in PBS and passed through a $70 \mu \mathrm{m}$ cell strainer before to be counted and stored in liquid nitrogen using CryoMaxx medium (PAA Laboratories). ${ }^{61}$ PBMCs and TILs were quickly thawed in culture media (RPMI1640 [Gibco, Invitrogen] $+10 \%$ human $\mathrm{AB}^{+}$serum [Institut de Biotechnologies Jacques Boy] $+2 \mathrm{mM}$ L-Glutamine $+1 \%$ sodium/pyruvate $+1 \%$ penicillin/streptomycin [all from Gibco, Invitrogen]), washed, counted and seeded at $0.3 \times 10^{6} / \mathrm{mL}$ per well in 48 well-plate in culture medium and incubated with or without Roferon ${ }^{\circledR}(1000 \mathrm{UI} / \mathrm{mL}$, Roche Pharma) and LPS (10 ng/mL, Sigma). After 24-60 h of incubation, cells were harvested, washed, immediately resuspended in $\mathrm{RLT}+$ Buffer and stored at $-80^{\circ} \mathrm{C}$ until RNA extraction and quantification.

\section{Gene expression analyses}

Total RNA extraction and genomic DNA removal were performed with the RNeasy Mini kit (Qiagen), following manufacturer's recommendations and quantified using a NanoDrop ${ }^{T M}$ Spectrophotometer (Thermo Fisher Scientific). RNA was either reverse transcribed into CDNA or RT-PCR amplifications were performed using TaqMan ${ }^{\mathrm{TM}}$ RNA-to-CT ${ }^{\mathrm{TM}}$ 1-Step Kit (ThermoFisher Scientific) Reverse transcription. A maximum of $1 \mu \mathrm{g}$ of RNA was reverse transcribed into cDNA with a mix comprising SuperScript III Reverse Transcriptase (Life Technologies), RNaseOUT ${ }^{\mathrm{TM}}$ Recombinant Ribonuclease Inhibitor (Life Technologies), Random primers (Promega) and Deoxynucleoside Triphosphate Set, PCR grade (Roche Diagnostics). Quantitative gene expression assay. Expression of $\beta 2 M(\mathrm{Hs} 00187842 \mathrm{~m} 1)$, NOS2 (Hs01075529 m1), NOS3 (Hs01574665_m1) and PDL1 (Hs00204257_m1) (all from Life Technologies) were analyzed with TaqMan ${ }^{\circledR}$ Gene Expression Assay using the Universal Master Mix II. Amplifications were carried out using the following ramping profile: 1 cycle at $95^{\circ} \mathrm{C}$ for $10 \mathrm{~min}$, followed by 45 cycles of $95^{\circ} \mathrm{C}$ for $30 \mathrm{~s}, 60^{\circ} \mathrm{C}$ for $1 \mathrm{~min}$. 1-Step qRT-PCR. A maximum of $500 \mathrm{ng}$ of RNA was used in $25 \mu \mathrm{L}$ reaction volumes, using the same ramping profile as above with the addition of $1 \mathrm{cycle}$ at $48{ }^{\circ} \mathrm{C}$ for $15 \mathrm{~min}$ at the beginning of the amplification. All reactions were perfomed on a StepOnePlus ${ }^{\mathrm{TM}}$ or QuantStudio 3 Real-Time PCR System (Life Technologies). Quantitative RT-PCR data were normalized to the expression levels of the housekeeping genes $\beta 2 M$ by means of the $2^{-\Delta \Delta \mathrm{Ct}}$, taking into account the values obtained from the control conditions.

\section{Biospecimen collection at the MD Anderson}

(Andrews M.C. et al, manuscript in preparation) Wargo's team assembled a cohort of patients with metastatic melanoma receiving combination of ICB either on clinical trials or as standard of care (SOC) therapy between 01/01/2014 and 08/31/2017. Patients were excluded if insufficient data were available to determine radiographic responses. Patients were classified as "responders" ( $R$, being complete response + partial response) or "non-responders" (NR, being stable disease + progressive disease) based on their best overall response (BOR) to ICBs measured by RECIST v1.1. This cohort comprised patients with stage IV disease $(n=22,96 \%)$ or unresectable stage IIIC $(n=1,4 \%)$ and similar numbers of systemic therapy-naïve or pre-treated patients $(n=12$ (52\%) vs. 11 (48\%), respectively); notably, one third ( $n=7,30 \%)$ of patients had received prior pharmacologic immune-stimulating therapies ( $n=3$ IFN, $n=3$ IL-2, $n=3$ ipilimumab monotherapy, $n=3$ pembrolizumab monotherapy, $n=1$ atezolizumab monotherapy). Available pre-treatment tumor samples were identified and retrieved for correlative molecular analyses. Gene expression profiling was performed using a custom-designed 795-gene codeset as previously described (Supplementary information, Table S3). ${ }^{62}$ Within-cohort housekeeping genes were selected by identifying the two most stably-expressed genes based on genewise CV within each of the upper, lower, and interquartile ranges of genes (lower: IFIT1B, IL20; interquartile: IKBKG, TRIM5; upper: ACTB, ZC3HAV1). Differential gene expression analyses were performed on count data using the R package NanoStringDiff $(v 1.10 .0){ }^{63}$ For statistical significance, fold change of $>2$ or $<$ 0.5 (calculated as the ratio of average gene expression intensity in $\mathrm{R}$ versus NR) with an FDR adjusted $p<0.10$ was used. Normalized data (for visualizations) were generated from raw count data using the variance stabilizing normalization implemented in the $R$ package NanoStringNorm (v1.2.1). ${ }^{64}$

\section{Human tumor histology}

Immunohistochemistry, Scanning and Analyzing of Systems. Composition and cellularity were assessed using a hematoxylin, eosin \& safran stain (H\&E). The staining of CD68 and NOS2 were performed on an automated immunostainer (The BenchMark ULTRA, Ventana, Gustave Roussy). Heat-induced antigen retrieval in EDTA buffer ( $\mathrm{pH}$ 8.0) was performed respectively for $32 \mathrm{~min}$ (CD68) and for $64 \mathrm{~min}(\mathrm{NOS2})$ at $95^{\circ} \mathrm{C}$. The mouse monoclonal anti-human CD68 antibody (clone PG-M1, $30 \mathrm{mg} / \mathrm{mL}$, \#M0876, Dako Agilent) was diluted 1:200 in antibody diluent (Zytomed) and the slides were incubated for $1 \mathrm{~h}$ at room temperature. The mouse monoclonal anti-human NOS2 antibody $(4.8 \mathrm{mg} / \mathrm{mL}$, \#DMAB5712RH, Diagnostic Creative) was diluted 1:240 in antibody diluent (Zytomed) and the slides were incubated for $1 \mathrm{~h}$ at $37^{\circ} \mathrm{C}$. The biotin-free alkaline phosphatase system of detection technique with Fast red as chromogen was applied (ultraView Universal Alkaline Phosphatase Red Detection Kit, Ventana). The slides were also counterstained by Hematoxylin kit (Ventana). Images displayed in the figures were taken with Zeiss Axio Scan. Z2 (primary objective_20/0.4, ocular_objective_10) and exported from the Zen 2 lite software as TIFF images. Some images were processed with using algorithm developed in Visiopharm Integrator System (VIS) (Visiopharm A/S, Denmark). WSI with the stains $\mathrm{H} \& \mathrm{E}, \mathrm{CD} 68$ and NOS2 were saved and stacked thanks to the Visiopharm module TISSUEalign. Intratumoral and peritumoral regions were then defined manually on the $\mathrm{H} \& \mathrm{E}$ layer. After CD68 positive cells were identified and counted in those regions, a cell density map (heatmap) was computed and regions with the highest density of CD68 positive cells (hot spots) were automatically identified. Then the density of NOS2 positive surface was quantified on these hotspots of CD68 positive cells. 4 patients out of 11 were not able to be quantified and, therefore, were excluded from the final analysis represented in Supplementary information, Fig. S10. Patient's characteristics of the total cohort can be found in Supplementary information, Table S2.

\section{Statistical analyses}

Data analyses and representations were performed either with the R software (http://www.R-project.org/) or Prism 5 (GraphPad, San Diego, CA, USA). Statistical analyses gathering more than two groups were performed using ANOVA followed with pairwise comparisons with Bonferroni adjustments. Otherwise, for two groups, statistical analyses were performed using the unpaired $t$ test or Wilcoxon matched-pairs signed rank test. Outliers within a given distribution were tested using Grubbs' test (https:// graphpad.com/quickcalcs/Grubbs1.cfm) with a threshold at $p<$ 0.05. Tumor growth experiments were analyzed with the TumGrowth software (https://kroemerlab.shinyapps.io/TumGrowth/), ${ }^{65}$ with default settings at the exception of the original tumor measurements that were log transformed before linear mixedeffect modeling. Cox proportional hazards modelling were applied when assessing the impact of the treatment on mice survival. Unless stated, all $p$-values are reported after Bonferroni correction when the question is addressing more than 2 experimental conditions. $p$-values were two-sided with $95 \%$ confidence intervals and were considered significant when $p<0.05$. Symbol significance: ${ }^{*} p<0.05,{ }^{* *} p<0.01,{ }^{* * *} p<0.001$. 


\section{ACKNOWLEDGEMENTS}

We would like to particularly thanks the animal facility, the Flowcytometry and Pathology Platforms of Gustave Roussy, especially Dr. Patrick Gonin, Laure Touchard, Philippe Rameau, and Olivia Bawa. We thank the Professors Antoine Durrbach and Gilles Uzé for providing Abatacept and Ifnar $1^{-1-}$ mice, respectively. We are grateful to Sebastien Jacques from the Genomic platform, Université Paris Descartes, Institut Cochin, Paris, France for his help in the microarray experiment. We thank Vichnou Poirier-Colame for her help and expertise in histology. This work was supported by Institut National du Cancer (INCa), ANR, Ligue contre le cancer and Swiss Bridge Foundation, ISREC Foundation, LABEX Oncolmmunology, la direction générale de I'offre de soins (DGOS), Université Paris-Sud, PIA2 TORINO-LUMIERE, Chancelerie des universités de Paris (Legs Poix), Fondation pour la Recherche Médicale (FRM); a donation by Elior; European Research Area Network on Cardiovascular Diseases (ERACVD, MINOTAUR); Gustave Roussy Odyssea, the European Union Horizon 2020 Project Oncobiome; Fondation Carrefour; Inserm (HTE); Institut Universitaire de France; LeDucq Foundation; the LabEx Immuno-Oncology; the RHU Torino Lumière; the Seerave Foundation; the SIRIC Stratified Oncology Cell DNA Repair and Tumor Immune Elimination (SOCRATE); and the SIRIC Cancer Research and Personalized Medicine (CARPEM). N.J. received a fellowship from Fondation ARC pour la recherche sur le cancer and is supported by grant 1163990 awarded through the 2018 Prioritydriven Collaborative Cancer Research Scheme and co-funded by Cancer Australia and Cure Cancer. M.C.A. is supported by a National Health and Medical Research Council (NHMRC) of Australia CJ Martin Early Career Fellowship (1148680). This research was supported by the generous philanthropic contributions to The University of Texas MD Anderson Cancer Center Moon Shots Program ${ }^{\mathrm{TM}}$ from the Lyda Hill Foundation and utilized platform assistance from the Cancer Genomics Laboratory; from the Dr. Miriam and Sheldon G. Adelson Medical Research Foundation, and the AIM at Melanoma Foundation. This work was supported by grants from Italian Miinistry of Health through "Ricerca Corrente". G.M. is funded by Istitutional "Ricerca Corrente". M.J.S. was supported by a NHMRC of Australia Senior Principal Research Fellowship (1078671) and Program Grant (1132519). V.B. and S.U. are supported by the Italian Association for Cancer Research (AIRC 12182, 21509 and 18603). S.F.N. was supported by an Australia NHMRC CJ Martin Fellowship (1111469).

\section{AUTHOR CONTRIBUTIONS}

L.Z. and G.K. supervised the study. N.J. and T.Y. performed most of the experiments with the help of M.P.R., G.F., C.P.M.D., S.B., M.V., R.D., M.M., S.U., I.M., V.P.C. and C.K. N.J., T.Y., M.P.R., C.P.M.D., S.B., M.M., D.P.E., G.S., S.U., I.M., P.O. and C.K. analyzed the data. N.J., T.Y., M.P.R., C.P.M.D., S.B., M.M., D.P.E., G.S., S.U., I.M. and K.C. interpreted the data. S.F.N., A.M., A.P.-B., A.M.E., G.M., P.A.A., A.S., D.S., M.J.S. provided scientific advice, patient samples, clinical information and experimental tools. M.C.A., J.W., P.-O.G. and V.G. provided the anti-PD-1 and anti-CTLA-4-treated clinical cohort and performed analyses. N.J. and L.Z. wrote the initial paper. All authors reviewed, edited and approved the final version of the manuscript.

\section{ADDITIONAL INFORMATION}

Supplementary information accompanies this paper at https://doi.org/10.1038/ s41422-019-0224-x.

Competing interests: G.K. reports grants and personal fees from Bayer Healthcare, grants from Genentech, grants from Glaxo Smyth Kline, grants and personal fees from Lytix Pharma, grants from PharmaMar, grants from Sotio, grants from Vasculox, other from Bristol Myers Squibb Foundation France, other from everlmmune, other from Samsara Therapeutics, outside the submitted work. L.Z. has the following financial relationships to disclose, none of which deals with the submitted work: Scientific advisory boards at Lytix Pharma, EpiVax, NeoVax and Tusk Pharma; Administrative board: Transgene; Grant/Research support from Glaxo Smith Kline, Merus, Tusk Roche and Incyte; Founder and shareholder: everlmmune. M.C.A. reports honorarium, travel support and advisory board participation from Merck, unrelated to the current work. J.W. and V.G. are inventors on a US patent application (PCT/US17/ 53.717) submitted by the University of Texas MD Anderson Cancer Center that covers methods to enhance immune checkpoint blockade responses by modulating the microbiome, unrelated to this work submitted. J.W. reports compensation for speaker's bureau and honoraria from Imedex, Dava Oncology, Omniprex, Illumina, Gilead, Medlmmune and Bristol-Myers Squibb; consultant/advisory board membership for AstraZeneca, Merck, Biothera Pharmaceuticals and Microbiome DX; consultant/advisory board membership and research support from GlaxoSmithKline, Roche/Genentech, Bristol-Myers Squibb, and Novartis. V.G. reports consultancy fees from MicrobiomeDX and ExpertConnect, and honoraria from ExcelCME and Kansas Society of Clinical Oncology. M.J.S. has scientific research agreements with Bristol Myers Squibb and Tizona Therapeutics and is an advisory board member for Compass Therapeutics and Tizona Therapeutics. A.M.E. declares receipt over the last 2 years of Honoraria for Scientific Advisory Boards and Data Monitoring Safety Board from the following companies: BMS, GSK, Incyte, IO Biotech, ISA-Pharmaeuticals, Medlmmune, Merck-Serono, MSD, Novartis, Pfizer, Sanofi, Sellas, SkylineDx. P.A.A. has/had a consulting or advisory role for BMS, Roche-Genentech, MSD, Array, Novartis, Merck Serono, Pierre Fabre, Incyte, Genmab, Newlink Genetics, Medimmune, AstraZeneca, Syndax, Sun Pharma, Sanofi, Idera, Ultimovacs. Sandoz, Immunocore. He also received research funds from BMS, Roche-Genentech, Array, and travel support from MSD. V.B. reports advisory board participation from ITeos Therapeutics Sa, Tusk Therapeutics Ltd, lo Biotech ApS, Xios Therapeutics, Codiak BioSciences. Over the last 5 years, J.-C.S. has received consultancy fees from AstraZeneca, Astex, Clovis, GSK, GamaMabs, Lilly, MSD, Mission Therapeutics, Merus, Pfizer, PharmaMar, Pierre Fabre, Roche/Genentech, Sanofi, Servier, Symphogen, and Takeda. He has been a full-time employee of Medlmmune since September 2017. He is a shareholder of AstraZeneca and Gritstone. L.V. reports personal fees from Adaptherapy, personal fees from Pierre-Fabre, grants from Bristol-Myers Squibb, outside the submitted work. The remaining authors declare that they have no conflict of interest.

\section{REFERENCES}

1. Hanahan, D. \& Weinberg, R. A. Hallmarks of cancer: the next generation. Cell 144, 646-674 (2011).

2. Smyth, M. J., Ngiow, S. F., Ribas, A. \& Teng, M. W. Combination cancer immunotherapies tailored to the tumour microenvironment. Nat. Rev. Clin. Oncol. 13, 143-158 (2016).

3. Zitvogel, L., Tesniere, A. \& Kroemer, G. Cancer despite immunosurveillance: immunoselection and immunosubversion. Nat. Rev. Immunol. 6, 715-727 (2006).

4. Le, D. T. et al. PD-1 blockade in tumors with mismatch-repair deficiency. N. Engl. J. Med. 372, 2509-2520 (2015).

5. Riaz, N. et al. Recurrent SERPINB3 and SERPINB4 mutations in patients who respond to anti-CTLA4 immunotherapy. Nat. Genet. 48, 1327-1329 (2016).

6. Rizvi, N. A. et al. Cancer immunology. Mutational landscape determines sensitivity to PD-1 blockade in non-small cell lung cancer. Science 348, 124-128 (2015)

7. Schumacher, T. N. \& Schreiber, R. D. Neoantigens in cancer immunotherapy. Science 348, 69-74 (2015).

8. Van Allen, E. M. et al. Genomic correlates of response to CTLA-4 blockade in metastatic melanoma. Science 350, 207-211 (2015).

9. Sharma, P. \& Allison, J. P. Immune checkpoint targeting in cancer therapy: toward combination strategies with curative potential. Cell 161, 205-214 (2015).

10. Sharma, P. \& Allison, J. P. The future of immune checkpoint therapy. Science $\mathbf{3 4 8 ,}$ 56-61 (2015).

11. Restifo, N. P., Dudley, M. E. \& Rosenberg, S. A. Adoptive immunotherapy for cancer: harnessing the T cell response. Nat. Rev. Immunol. 12, 269-281 (2012).

12. Borghaei, $\mathrm{H}$. et al. Nivolumab versus docetaxel in advanced nonsquamous nonsmall-cell lung cancer. N. Engl. J. Med. 373, 1627-1639 (2015).

13. Nghiem, P. T. et al. PD-1 Blockade with pembrolizumab in advanced merkel-cell carcinoma. N. Engl. J. Med. 374, 2542-2552 (2016).

14. Robert, C. et al. Ipilimumab plus dacarbazine for previously untreated metastatic melanoma. N. Engl. J. Med. 364, 2517-2526 (2011).

15. Rosenberg, J. E. et al. Atezolizumab in patients with locally advanced and metastatic urothelial carcinoma who have progressed following treatment with platinum-based chemotherapy: a single-arm, multicentre, phase 2 trial. Lancet 387, 1909-1920 (2016).

16. Hirsch, L., Zitvogel, L., Eggermont, A. \& Marabelle, A. PD-Loma: a cancer entity with a shared sensitivity to the PD-1/PD-L1 pathway blockade. Br J Cancer. 120, 3-5 (2019).

17. Hargadon, K. M., Johnson, C. E. \& Williams, C. J. Immune checkpoint blockade therapy for cancer: an overview of FDA-approved immune checkpoint inhibitors. Int. Immunopharmacol. 62, 29-39 (2018).

18. Pardoll, D. Cancer and the immune system: basic concepts and targets for intervention. Semin. Oncol. 42, 523-538 (2015).

19. Topalian, S. L. et al. Safety, activity, and immune correlates of anti-PD-1 antibody in cancer. N. Engl. J. Med. 366, 2443-2454 (2012).

20. Gandhi, L. et al. Pembrolizumab plus chemotherapy in metastatic non-small-cell lung cancer. N. Engl. J. Med. 378, 2078-2092 (2018).

21. Spranger, S., Bao, R. \& Gajewski, T. F. Melanoma-intrinsic beta-catenin signalling prevents anti-tumour immunity. Nature 523, 231-235 (2015).

22. Neubert, N. J. et al. T cell-induced CSF1 promotes melanoma resistance to PD1 blockade. Sci. Transl. Med. 10, eaan3311 (2018).

23. Gao, J. et al. Loss of IFN-gamma pathway genes in tumor cells as a mechanism of resistance to anti-ctla-4 therapy. Cell 167, 397-404 e399 (2016).

24. Koyama, S. et al. Adaptive resistance to therapeutic PD-1 blockade is associated with upregulation of alternative immune checkpoints. Nat. Commun. 7, 10501 (2016). 
25. Ribas, A. et al. Association of pembrolizumab with tumor response and survival among patients with advanced melanoma. JAMA 315, 1600-1609 (2016).

26. Zaretsky, J. M. et al. Mutations associated with acquired resistance to pd-1 blockade in melanoma. N. Engl. J. Med. 375, 819-829 (2016).

27. Sade-Feldman, M. et al. Resistance to checkpoint blockade therapy through inactivation of antigen presentation. Nat. Commun. 8, 1136 (2017).

28. Khong, H. T. \& Restifo, N. P. Natural selection of tumor variants in the generation of "tumor escape" phenotypes. Nat. Immunol. 3, 999-1005 (2002).

29. Restifo, N. P. et al. Loss of functional beta 2-microglobulin in metastatic melanomas from five patients receiving immunotherapy. J. Natl. Cancer Inst. 88, 100-108 (1996).

30. Pardoll, D. M. The blockade of immune checkpoints in cancer immunotherapy. Nat. Rev. Cancer 12, 252-264 (2012).

31. Taube, J. M. et al. Colocalization of inflammatory response with B7-h1 expression in human melanocytic lesions supports an adaptive resistance mechanism of immune escape. Sci. Transl. Med. 4, 127ra137 (2012).

32. Landsberg, J. et al. Melanomas resist T-cell therapy through inflammationinduced reversible dedifferentiation. Nature 490, 412-416 (2012).

33. Ngiow, S. F. et al. A threshold level of intratumor CD8+ T-cell PD1 expression dictates therapeutic response to Anti-PD1. Cancer Res. 75, 3800-3811 (2015).

34. Gajewski, T. F. \& Corrales, L. New perspectives on type I IFNs in cancer. Cytokine Growth Factor Rev. 26, 175-178 (2015).

35. Rodriguez-Ruiz, M. E. et al. Abscopal effects of radiotherapy are enhanced by combined immunostimulatory mabs and are dependent on cd8 t cells and crosspriming. Cancer Res. 76, 5994-6005 (2016).

36. Smyth, M. J., Dunn, G. P. \& Schreiber, R. D. Cancer immunosurveillance and immunoediting: the roles of immunity in suppressing tumor development and shaping tumor immunogenicity. Adv. Immunol. 90, 1-50 (2006).

37. Wang, $X$. et al. Suppression of type $i$ ifn signaling in tumors mediates resistance to anti-pd-1 treatment that can be overcome by radiotherapy. Cancer Res. 77, 839-850 (2017).

38. Sistigu, A. et al. Cancer cell-autonomous contribution of type I interferon signaling to the efficacy of chemotherapy. Nat. Med. 20, 1301-1309 (2014).

39. Dunn, G. P. et al. A critical function for type I interferons in cancer immunoediting. Nat. Immunol. 6, 722-729 (2005).

40. Bronte, V. et al. IL-4-induced arginase 1 suppresses alloreactive T cells in tumorbearing mice. J. Immunol. 170, 270-278 (2003).

41. Dussurget, O., Bierne, H. \& Cossart, P. The bacterial pathogen Listeria monocytogenes and the interferon family: type I, type II and type III interferons. Front. Cell. Infect. Microbiol. 4, 50 (2014).

42. Mondanelli, G., Ugel, S., Grohmann, U. \& Bronte, V. The immune regulation in cancer by the amino acid metabolizing enzymes ARG and IDO. Curr. Opin. Pharm. 35, 30-39 (2017).

43. Rodriguez, P. C. et al. Arginase I production in the tumor microenvironment by mature myeloid cells inhibits T-cell receptor expression and antigen-specific Tcell responses. Cancer Res. 64, 5839-5849 (2004).

44. Rodriguez, P. C. \& Ochoa, A. C. Arginine regulation by myeloid derived suppressor cells and tolerance in cancer: mechanisms and therapeutic perspectives. Immunol. Rev. 222, 180-191 (2008).

45. Modolell, M., Corraliza, I. M., Link, F., Soler, G. \& Eichmann, K. Reciprocal regulation of the nitric oxide synthase/arginase balance in mouse bone marrow-derived macrophages by TH1 and TH2 cytokines. Eur. J. Immunol. 25, 1101-1104 (1995).

46. Verbeke, $H$. et al. Isotypic neutralizing antibodies against mouse GCP-2/CXCL6 inhibit melanoma growth and metastasis. Cancer Lett. 302, 54-62 (2011).

47. Benci, J. L. et al. Tumor interferon signaling regulates a multigenic resistance program to immune checkpoint blockade. Cell 167, 1540-1554 e1512 (2016).

48. Hugo, W. et al. Genomic and transcriptomic features of response to anti-PD-1 therapy in metastatic melanoma. Cell 165, 35-44 (2016).

49. Hugo, W. et al. Non-genomic and immune evolution of melanoma acquiring MAPKi resistance. Cell 162, 1271-1285 (2015).
50. Vannini, F., Kashfi, K. \& Nath, N. The dual role of iNOS in cancer. Redox Biol. 6 334-343 (2015).

51. Girotti, A. W., Fahey, J. M. \& Korytowski, W. Multiple means by which nitric oxide can antagonize photodynamic therapy. Curr. Med. Chem. 23, 2754-2769 (2016).

52. Lu, G. et al. Myeloid cell-derived inducible nitric oxide synthase suppresses M1 macrophage polarization. Nat. Commun. 6, 6676 (2015).

53. Farlik, M. et al. Nonconventional initiation complex assembly by STAT and NFkappaB transcription factors regulates nitric oxide synthase expression. Immunity 33, 25-34 (2010)

54. Diefenbach, A. et al. Type 1 interferon (IFNalpha/beta) and type 2 nitric oxide synthase regulate the innate immune response to a protozoan parasite. Immunity 8, 77-87 (1998).

55. Mattner, J. et al. Regulation of type 2 nitric oxide synthase by type 1 interferons in macrophages infected with Leishmania major. Eur. J. Immunol. 30, 2257-2267 (2000).

56. Moreira-Teixeira, L. et al. Type I IFN inhibits alternative macrophage activation during mycobacterium tuberculosis infection and leads to enhanced protection in the absence of IFN-gamma signaling. J. Immunol. 197, 4714-4726 (2016).

57. Yamazaki, $\mathrm{T}$. et al. Blockade of $\mathrm{B} 7-\mathrm{H} 1$ on macrophages suppresses $\mathrm{CD} 4+\mathrm{T}$ cell proliferation by augmenting IFN-gamma-induced nitric oxide production. J. Immunol. 175, 1586-1592 (2005).

58. Marigo, I. et al. T cell cancer therapy requires CD40-CD40L activation of tumor necrosis factor and inducible nitric-oxide-synthase-producing dendritic cells. Cancer Cell 30, 377-390 (2016).

59. Gaj, T., Guo, J., Kato, Y., Sirk, S. J. \& Barbas, C. F. 3rd Targeted gene knockout by direct delivery of zinc-finger nuclease proteins. Nat. Methods 9, 805-807 (2012).

60. Ritchie, M. E. et al limma powers differential expression analyses for RNAsequencing and microarray studies. Nucleic Acids Res. 43, e47 (2015).

61. Jacquelot, N. et al. Immunophenotyping of stage III melanoma reveals parameters associated with patient prognosis. J. Invest. Dermatol. 136, 994-1001 (2016).

62. Chen, P. L. et al. Analysis of immune signatures in longitudinal tumor samples yields insight into biomarkers of response and mechanisms of resistance to immune checkpoint blockade. Cancer Discov. 6, 827-837 (2016).

63. Wang, H., Zhai, T. \& Wang, C. NanoStringDiff: differential expression analysis of NanoString nCounter data. R package version 1.12 .0 (2018).

64. Waggott, D. et al. NanoStringNorm: an extensible $R$ package for the preprocessing of NanoString mRNA and miRNA data. Bioinformatics 28, 1546-1548 (2012).

65. Enot, D. P., Vacchelli, E., Jacquelot, N., Zitvogel, L. \& Kroemer, G. TumGrowth: an open-access web tool for the statistical analysis of tumor growth curves. Oncoimmunology 7, e1462431 (2018).

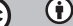

Open Access This article is licensed under a Creative Commons Attribution 4.0 International License, which permits use, sharing, adaptation, distribution and reproduction in any medium or format, as long as you give appropriate credit to the original author(s) and the source, provide a link to the Creative Commons license, and indicate if changes were made. The images or other third party material in this article are included in the article's Creative Commons license, unless indicated otherwise in a credit line to the material. If material is not included in the article's Creative Commons license and your intended use is not permitted by statutory regulation or exceeds the permitted use, you will need to obtain permission directly from the copyright holder. To view a copy of this license, visit http://creativecommons. org/licenses/by/4.0/.

(c) The Author(s) 2019 